\title{
Permutations Avoiding Certain Partially-ordered Patterns
}

\author{
Kai Ting Keshia Yap \\ Department of Mathematics \& Statistics \\ Queens University \\ 48 University Ave. Jeffery Hall \\ Kingston, ON Canada K7L 3N6 \\ yap.keshia@gmail.com \\ David Wehlau* Imed Zaguia ${ }^{\dagger}$ \\ Department of Mathematics \& Computer Science \\ Royal Military College of Canada \\ P.O. Box 17000, Station Forces \\ Kingston, Ontario, Canada K7K 7B4 \\ \{David.Wehlau, Imed.Zaguia\}@rmc.ca
}

Submitted: Jan 28, 2021; Accepted: Mar 25, 2021; Published: Jul 16, 2021

(C) The authors. Released under the CC BY-ND license (International 4.0).

\begin{abstract}
A permutation $\pi$ contains a pattern $\sigma$ if and only if there is a subsequence in $\pi$ with its letters in the same relative order as those in $\sigma$. Partially ordered patterns (POPs) provide a convenient way to denote patterns in which the relative order of some of the letters does not matter. This paper elucidates connections between the avoidance sets of a few POPs with other combinatorial objects, directly answering five open questions posed by Gao and Kitaev in 2019. This was done by thoroughly analysing the avoidance sets and developing recursive algorithms to derive these sets and their corresponding combinatorial objects in parallel, which yielded natural bijections. We also analysed an avoidance set whose simple permutations are enumerated by the Fibonacci numbers and derived an algorithm to obtain them recursively.
\end{abstract}

Mathematics Subject Classifications: 05A05, 05A15

\section{Introduction}

This paper elucidates connections between the avoidance sets of a few Partially Ordered Patterns (POPs) with other combinatorial objects, directly answering five open questions

*Partially supported by NSERC

${ }^{\dagger}$ Partially supported by NSERC. Corresponding author. 
posed by Gao and Kitaev [5]. Results in this article appeared in the first author's MSC dissertation.

We write $[n]$ to denote the set of integers $\{1,2, \ldots, n\}$ for $n \geqslant 1$ and $[i, j]$, with $i \leqslant j$, to denote the set of integers $\{i, i+1, \ldots, j\}$. A permutation is a bijection from $[n]$ to itself for some $n \geqslant 1$. We call such a permutation an $n$-permutation and typically denote it by $\pi=\pi_{1} \pi_{2} \cdots \pi_{n}$, where $\pi_{i}=\pi(i)$. We say that its length or size (denoted $|\pi|$ ) is $n$. We write $S_{n}$ to denote the set of all $n$-permutations. We denote the size of any set $S$ by $\# S$ or $|S|$.

\section{$1.1 \quad$ Background}

A permutation $\pi$ contains a pattern $\sigma$ if and only if there is a subsequence in $\pi$ (of the same length as $\sigma$ ) with its letters in the same relative order as those in $\sigma$. For instance, the pattern 312 occurs in 42531 (as the subsequence 423), but not in 132465. The permutations that avoid a pattern or a set of patterns make up an avoidance set. Avoidance sets have been studied extensively and research in this area has important applications to numerous fields. Examples include sorting devices in theoretical computer science, Schubert varieties and Kazhdan-Lusztig polynomials, statistical mechanics, the tandem duplication-random loss model in computational biology and bijective combinatorics (see [6] and references therein).

A partially ordered pattern (abbreviated POP) is a partially ordered set (poset) that generalizes the notion of a pattern when we are not concerned with the relative order of some of its letters, and therefore may represent multiple patterns. Specifically, a POP is a poset with $n$ elements labelled $1,2, \ldots, n$, for some $n \geqslant 1$. For any pattern that the POP represents, the partial order of the elements stipulates the relative order of letters in the pattern, where the labels of the elements indicate the positional order of these letters.

For example, the POP $p=S_{2} S_{4}^{1}$ represents all the patterns of length 4 whose first element is larger than the third element. That is, $p$ represents the twelve patterns

2314, 2413, 3124, 3421, 3214, 3412, 4213, 4312, 4123, 4321, 4132 and 4231.

A POP may represent a single pattern. For example, the pattern 3241 represented as a POP is the chain of four elements labelled 1, 2, 3 and 4 with the order $4<2<1<3$. Note that 3241 is the permutation inverse of 4213, and this is not a coincidence.

A permutation contains a POP if and only if it contains at least one of the patterns represented by that POP. Otherwise, it avoids the POP. For example, the permutation 3472615 contains 21 occurrences of the POP $p$ (defined above) whereas 132456 avoids $p$.

\subsection{Motivation and structure}

Enumerating the permutations of different lengths in the avoidance set of a pattern or set of patterns and finding one-to-one correspondences to well-known combinatorial objects is a topic of great interest. Several classical combinatorial objects may be related to a single avoidance set, and finding these connections would allow us to understand seemingly 
disparate objects under a common framework [6]. With the aid of a computer software, Gao and Kitaev [5] conducted a systematic search of connections between sequences in The Online Encyclopedia of Integer Sequences (OEIS) [9] and the enumeration of permutations avoiding POPs with 4 or 5 elements. They observed connections to 38 sequences in OEIS and listed 15 combinatorial objects with which potentially interesting bijections might occur with the avoidance sets of certain POPs (see Tables 6 and 7 of their paper).

The goal of this paper is to find as many bijections as possible between the pairs of objects in ways that are meaningful. With the help of an interactive software PermLab [1], we successfully construct nontrivial bijections for five of these pairs and find generalizations whenever possible. We list the objects in Table 1 and discuss the bijections in Sections 2, 3, 4 and 5. One bijection (discussed in Section 3.2) emerges directly from the original proof of the enumeration of ground-state juggling sequences by Chung and Graham [4]. For each of the remaining four bijections, we first realize that both sets in the corresponding pair can be partitioned into subsets of corresponding sizes. This allows us to construct similar recursive algorithms that can build the sets in parallel, which in turn yield (one or many) bijections that could be constructed directly and explicitly. Thus, we end up with a thorough understanding of the permutations that avoid each POP and of the corresponding combinatorial objects.

During our analysis, we discovered a set of patterns that are avoided by infinitely many simple permutations (to be defined in Section 1.3), which are, in fact, enumerated by a translation of the well-known Fibonacci sequence. We construct an algorithm that allows one to obtain this set of permutations recursively and prove this in Section 6 . Section 1.3 defines all the relevant terms and concepts in detail and Section 7 summarises our research and lists possible avenues of further research.

\subsection{Preliminaries}

For an $n$-permutation $\pi$, we say that $\pi_{i_{1}} \pi_{i_{2}} \cdots \pi_{i_{k}}$ is a subsequence of $\pi$ if and only if $1 \leqslant i_{1}<i_{2}<\cdots i_{k} \leqslant n$ and $k \in[n]$. For an $n$-permutation $\pi$ and any $1 \leqslant i, j \leqslant n$, the contiguous substring $\pi_{i} \pi_{i+1} \cdots \pi_{j}$ is called a factor of $\pi$. We denote $\pi_{i} \pi_{i+1} \cdots \pi_{j}$ simply as $\pi_{[i, j]}$. Note that if $i=j$, then $\pi_{[i, j]}=\pi_{i}$ has length 1 and we call it a point, term or an element. If $i>j$ then $\pi_{[i, j]}$ has length 0 , and we say that it is empty. Let $\alpha=\pi_{\left[i_{1}, j_{1}\right]}$ and $\beta=\pi_{\left[i_{2}, j_{2}\right]}$ be non-empty factors of $\pi$. We write $\alpha<\beta$ if and only if $\pi_{\ell_{1}}<\pi_{\ell_{2}}$ for all $\ell_{1} \in\left[i_{1}, j_{1}\right]$ and $\ell_{2} \in\left[i_{2}, j_{2}\right]$.

Note that we may extend the definition of factors of permutations to factors of factors. If $\pi$ is a factor of size $n$ of a larger $m$-permutation $\zeta$, say $\pi=\zeta_{[i, j]}$ for some $1 \leqslant i \leqslant j \leqslant m$, then we use $\pi_{k}$ to denote $\zeta_{i+k-1}$ for any $k \in[n]$. Then $\pi_{[k, \ell]}=\zeta_{\left[i+k-1, i_{\ell}-1\right]}$ for any $1 \leqslant k \leqslant \ell \leqslant n$.

We say that a factor $\sigma=\pi_{[i, j]}$ (for some $1 \leqslant i \leqslant j \leqslant n$ ) of an $n$-permutation $\pi$ contains the point $x$, denoted as $x \in \sigma$, if and only if $\pi_{\ell}=x$ for some $\ell \in[i, j]$. Otherwise, we say that $\sigma$ does not contain the point $x$ and write $x \notin \sigma$. 


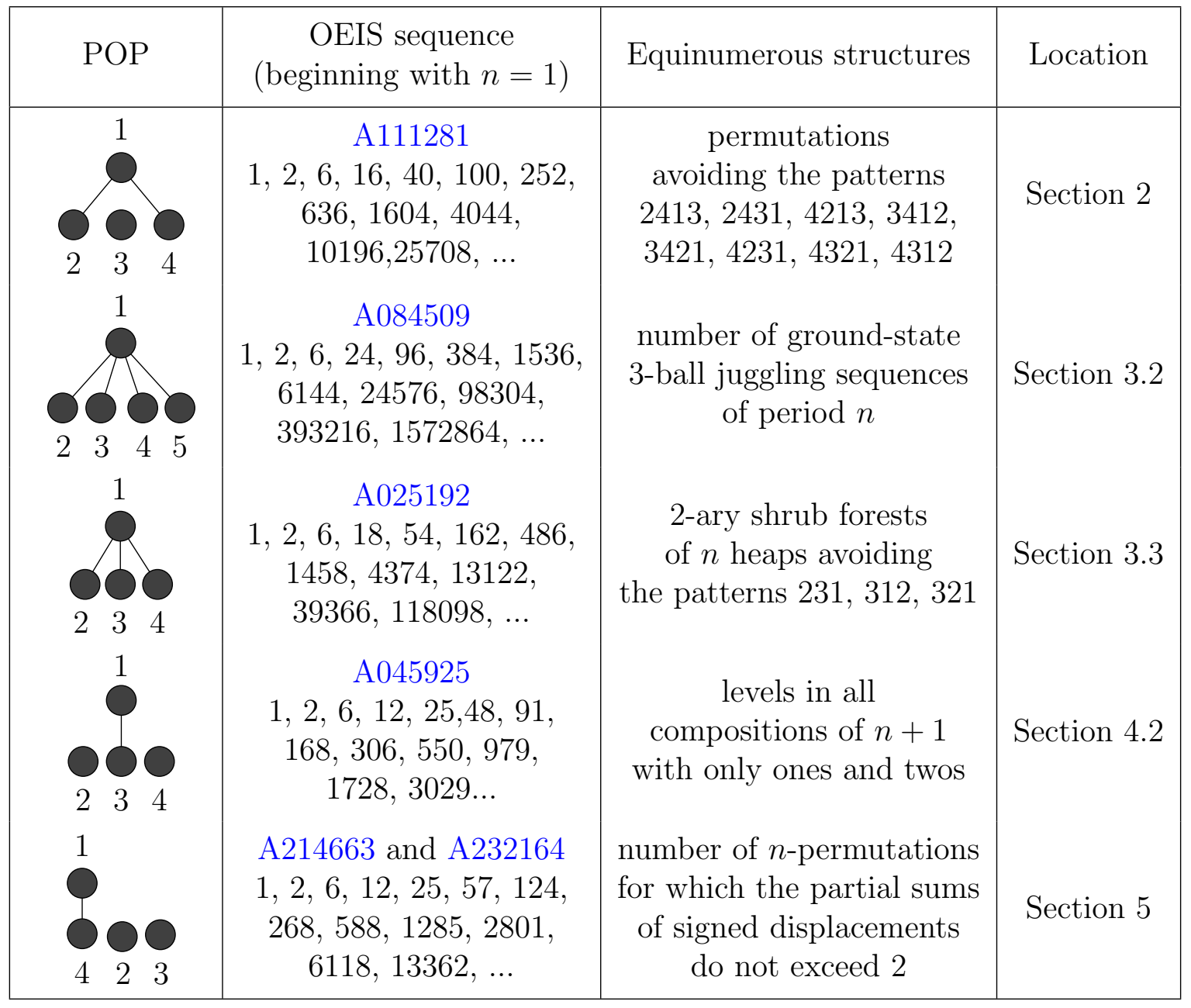

Table 1: List of POPs studied

For an $n$-permutation $\pi$, we say that a factor $\pi_{[i, j]}$ is an interval if and only if it contains exactly the numbers in a contiguous interval of $[n]$. That is, if and only if $\left\{\pi_{\ell} \mid \ell \in[i, j]\right\}=[s, t]$ for some $s, t \in[n]$. For example, the factor 2413 is an interval while the factor 241 is not an interval. An interval of an $n$-permutation is trivial if and only if its length is 0,1 or $n$.

Let $\pi_{i_{1}} \pi_{i_{2}} \cdots \pi_{i_{k}}$ be a subsequence of an $n$-permutation $\pi$. The reduced subsequence $\operatorname{red}\left(\pi_{i_{1}} \pi_{i_{2}} \cdots \pi_{i_{k}}\right)$ is defined as the $k$-permutation that is order-isomorphic to the subsequence. That is, $\operatorname{red}\left(\pi_{i_{1}} \pi_{i_{2}} \cdots \pi_{i_{k}}\right)=\sigma$ is the $k$-permutation where $\sigma_{s}<\sigma_{t}$ if and only if $\pi_{i_{s}}<\pi_{i_{t}}$ We say that $\sigma$ is the reduction of the subsequence $\pi_{i_{1}} \pi_{i_{2}} \cdots \pi_{i_{k}}$.

Example 1. Let $\pi=1435726$. Then the following statements are true:

(a) 1576 is a subsequence of $\pi$;

(b) $\operatorname{red}(1576)=1243$;

(c) $\pi_{[3,5]}=357$ and $\pi_{[6,7]}=26$ are factors of $\pi$;

(d) $\pi_{[2,4]}=435$ is an interval of $\pi$. 


\subsection{Simple permutations}

Definition 2. An $n$-permutation is simple if and only if all its intervals are trivial. That is, if and only if its intervals are all of length 0,1 or $n$.

Simple permutations were first considered in [8].

Definition 3. Let $\sigma$ be a $k$-permutation, and for $\ell \in[k]$, let $\alpha^{(\ell)}$ be a permutation of length $i_{\ell}$. We define the inflation of $\sigma$ by $\alpha^{(1)}, \alpha^{(2)}, \ldots, \alpha^{(k)}$ as the permutation

$$
\pi=\sigma\left[\alpha^{(1)}, \alpha^{(2)}, \ldots, \alpha^{(k)}\right]
$$

of length $n:=i_{1}+i_{2}+\cdots+i_{k}$ where given $s_{0}:=0, \ell \in[k], s_{\ell}:=i_{1}+i_{2}+\cdots+i_{\ell}$, the following hold:

- the factors $\pi_{\left[s_{\ell-1}+1, s_{\ell}\right]}$ are intervals;

- $\operatorname{red}\left(\pi_{\left[s_{\ell-1}+1, s_{\ell}\right]}\right)=\alpha^{(\ell)}$

- $\pi_{\left[s_{t-1}+1, s_{t}\right]}<\pi_{\left[s_{u-1}+1, s_{u}\right]}$ if and only if $\sigma_{t}<\sigma_{u}$.

We call $\sigma$ a quotient of $\pi$.

Example 4. The permutation 526314 is simple while the permutation 4215763 is not. The following statements are true:

(a) $4215763=3142[1,21,132,1]$. Note $4,21,576$ and 3 are intervals of 4215763 ;

(b) 3142 is a quotient of 4215763 ;

(c) 4215763 is an inflation of 3142 .

Proposition 5 (Albert and Atkinson [2]). Every permutation may be written as the inflation of a unique simple permutation. Moreover, if $\pi$ can be written as $\sigma\left[\alpha^{(1)}, \alpha^{(2)}, \ldots, \alpha^{(m)}\right]$ where $\sigma$ is simple and $m \geqslant 4$, then the $\alpha^{(i)} s$ are unique.

\subsection{Separable permutations}

Definition 6. Suppose $\pi$ and $\sigma$ are permutations of length $n$ and $m$ respectively. We define the direct sum (or simply, sum), using the operator $\oplus$, and the skew sum, using the operator $\ominus$, of $\pi$ and $\sigma$ as the permutations of length $m+n$ as follows:

$$
\pi \oplus \sigma=12[\pi, \sigma] \text { and } \pi \ominus \sigma=21[\pi, \sigma] .
$$

Definition 7. If a permutation is an inflation of 12 or 21, we call it sum decomposable and skew sum decomposable respectively. If a permutation is not sum decomposable we say it is sum indecomposable, and if it is not skew sum decomposable we say it is skew-sum indecomposable.

Definition 8. A permutation is separable if it can be obtained by repeatedly applying the $\oplus$ and $\ominus$ operations on the permutation 1 . 
Example 9. The permutation 587694231 is separable, since

$$
\begin{aligned}
587694231 & =14325 \ominus 4231 \\
& =(1 \oplus 3214) \ominus(1 \ominus 231) \\
& =(1 \oplus(321 \oplus 1) \ominus(1 \ominus(12 \ominus 1))) \\
& =(1 \oplus((1 \ominus 21) \oplus 1) \ominus(1 \ominus(12 \ominus 1))) \\
& =(1 \oplus((1 \ominus(1 \ominus 1)) \oplus 1) \ominus(1 \ominus((1 \oplus 1) \ominus 1))) .
\end{aligned}
$$

Example 10. All permutations of length 3 are separable. The only permutations of length 4 that are not separable are 2413 and 3142.

Theorem 11 (folklore). A permutation is separable if and only if it avoids 2413 and 3412 .

Proposition 12 (Albert and Atkinson [2]). If $\pi$ is an inflation of 12, say $\pi=12[\alpha, \beta]$, then $\alpha$ and $\beta$ are unique if $\alpha$ is sum indecomposable. The same holds with 12 replaced by 21 and "sum" replaced by "skew sum".

Corollary 13. All simple permutations of length at least 4 must contain the patterns 132, 213, 231 and 312.

Proof. It is clear that simple permutations are not separable, so by Theorem 11, they must contain at least one of 2413 or 3412 , both of which contain the four patterns of length three.

\subsection{Pattern/POP containment and avoidance}

Definition 14. A pattern is a permutation of length at least 2. We say that a permutation $\pi$ contains a pattern $p$ if and only if there exists some subsequence $\pi_{i_{1}} \pi_{i_{2}} \cdots \pi_{i_{k}}$ of $\pi$ where $\operatorname{red}\left(\pi_{i_{1}} \pi_{i_{2}} \cdots \pi_{i_{k}}\right)=p$. That is, $p_{j}<p_{\ell}$ if and only if $\pi_{i_{j}}<\pi_{i_{\ell}}$ for all $j, \ell \in[k]$. Otherwise, we say that $\pi$ avoids $p$. If $P$ is a set of patterns, we say that $\pi$ contains $P$ if $\pi$ contains any pattern in $P$. Otherwise we say that $\pi$ avoids $P$.

A partially ordered pattern generalizes the notion of a pattern whereby the order between certain elements do not have to be considered. We are left with a partial order on the elements, which we can represent using a labelled partially ordered set. Recall that a partial order is a binary relation $\leqslant$ over a set $P$ that is reflexive, antisymmetric and transitive. That is, for all $a, b, c \in P$, the following hold:

1. $a \leqslant a$ (reflexivity);

2. If $a \leqslant b$ and $b \leqslant a$ then $a=b$ (antisymmetry);

3. If $a \leqslant b$ and $b \leqslant c$ then $a<c$ (transitivity).

A set $P$ with a partial order $\leqslant$ is called a partially ordered set (poset), denoted $(P, \leqslant)$. We may write $b \geqslant a$ as an equivalent statement to $a \leqslant b$ for any $a, b \in P$. We write $a<b$ to mean that $a \leqslant b$ and that $a$ and $b$ are distinct. 
Definition 15. A partially ordered pattern (POP) $p$ of size $k$ is a poset with $k$ elements labelled $1,2, \ldots, k$. A POP can be expressed in one-line notation by indicating its size and the minimal set of relations that defines the respective poset.

Definition 16. An $n$-permutation $\pi$ contains such a POP $p$ if and only if $\pi$ has a subsequence $\pi_{i_{1}} \pi_{i_{2}} \cdots \pi_{i_{k}}$ such that $\pi_{i_{j}}<\pi_{i_{m}}$ if $j<m$ in the poset $P$. Otherwise, we say that $\pi$ avoids $p$.

Recall that a poset can be represented visually as a Hasse diagram. A Hasse diagram of a poset is a visual representation of its elements and covering relations. For a poset $(P, \leqslant)$, where $i$ and $j$ are elements of $P$, we say $j$ covers $i$ (notated $i \prec j$ ) if and only if $i<j$ and there does not exist any $k \in P$ such that both $i<k$ and $k<j$ hold. The binary relation $i \prec j$ is a covering relation and is illustrated by a line going upward from $i$ to $j$ in the Hasse diagram of $(P, \leqslant)$. The Hasse diagram of a poset uniquely determines its partial order.

A POP with $n$ elements can be represented by a Hasse diagram with its vertices labelled $1,2, \ldots, n$. Refer to the POP $p$ in Section 1.1 for the Hasse diagram representation of the POP of size 4 where $1>3$.

Definition 17. Let $P$ be a pattern, a set of patterns, or a POP. We denote $A v(P)$ as the set of permutations that avoid $P$ (called the avoidance set of $P$ ), and $A v_{n}(P)$ as the set of $n$-permutations that avoid $P$. That is, $A v_{n}(P):=A v(P) \cap S_{n}$.

Definition 18. Let $P_{1}$ and $P_{2}$ each be a set of patterns or a POP. We say that $P_{1}$ and $P_{2}$ are Wilf-equivalent if and only if $\left|A v_{n}\left(P_{1}\right)\right|=\left|A v_{n}\left(P_{2}\right)\right|$ for all $n \geqslant 1$.

It is not hard to check that containment is a partial order on any set of permutations. In the literature, sets of permutations which are closed downward under this order are called permutation classes, or sometimes just classes. That is, $\mathcal{C}$ is a permutation class if and only if for any $\pi \in \mathcal{C}$ and any $\sigma$ contained in $\pi$, we have $\sigma \in \mathcal{C}$. If a permutation $\pi$ avoids a pattern $p$, then every reduced subsequence of $\pi$ avoids $p$. In other words, every pattern contained in $\pi$ avoids $p$. Therefore $A v(p)$ and $A v_{n}(p)$ are permutation classes. The same is true if $p$ is a set of patterns or a POP.

Observe that if $k_{P}$ is the length of the shortest pattern in a set of patterns $P$, then all permutations of length less than $k_{P}$ avoid $P$. This means that $\left|A v_{n}(P)\right|=n$ ! for all $n<k_{P}$. Therefore it suffices to enumerate $A v_{n}(P)$ for $n \geqslant k_{P}$ for every POP or a set of patterns $P$ discussed in subsequent sections.

\subsection{Matrix representations of permutations}

Definition 19. For an $n$-permutation $\pi$, its permutation matrix is a binary $n \times n$ matrix, denoted $M(\pi)$ where

$$
M(\pi)_{n-i+1, j}=1 \Longleftrightarrow \pi_{j}=i .
$$

Moreover, its pattern matrix is an $n \times n$ matrix, denoted $M^{\prime}(\pi)$, where

$$
M^{\prime}(\pi)_{n-i+1, j}= \begin{cases}i & \text { if } \pi_{j}=i \\ 0 & \text { otherwise }\end{cases}
$$


We may refer to the non-zero entries in a permutation matrix or pattern matrix as points. Sometimes, we may omit displaying the 0s and the traditional brackets if no confusion would arise.

Example 20. Let $\pi=312$. Its permutation matrix is

$$
M(\pi)=\left(\begin{array}{lll}
1 & 0 & 0 \\
0 & 0 & 1 \\
0 & 1 & 0
\end{array}\right)=\begin{array}{lll}
1 & & \\
& & 1 \\
& 1 &
\end{array}
$$

and its pattern matrix is

$$
M^{\prime}(\pi)=\left(\begin{array}{lll}
3 & 0 & 0 \\
0 & 0 & 2 \\
0 & 1 & 0
\end{array}\right)=\begin{array}{lll}
3 & \\
& &
\end{array}
$$

Definition 21. The weight of a matrix is the number of non-zero entries it contains. We denote the weight of a matrix $A$ by $|A|$.

Example 22. The weight of the permutation matrix of $\pi$ is equal to the length of $\pi$. The weight of any column or row of a permutation matrix is 1 .

\subsection{Lattice matrices}

Definition 23. We call a matrix (or submatrix) void if it has no rows or no columns. A matrix (or submatrix) is trivial if its weight is 0 , and nontrivial otherwise. A void matrix is trivial, while a trivial matrix can be a void matrix or a zero matrix.

If we know that a permutation contains a certain pattern, it can be helpful to represent its permutation as a block matrix in order to better understand the permutation. We will show an example before stating formal definitions:

Suppose we know that a $n$-permutation $\pi$ contains the pattern $p:=312$. That is, there exist integers $i, j$ and $k$ where $1 \leqslant i<j<k \leqslant n$ and $\pi_{i} \pi_{j} \pi_{k}$ reduces to 312. The columns $i, j$ and $k$ partition the rest of the permutation matrix $M(\pi)$ into 4 (possibly trivial) blocks of columns, and the rows $\pi_{i}, \pi_{j}$ and $\pi_{k}$ partition the rest of the permutation matrix $M(\pi)$ into 4 (possibly trivial) blocks of rows. This gives rise to another representation of $M(\pi)$ as a $7 \times 7$ block matrix. In this representation we can find the $3 \times 3$ matrix $M(p)$ interwoven with a $4 \times 4$ block matrix $\left(\alpha_{i j}\right)_{i, j \in[4]}$ as depicted in Figure 1, with the following alterations:

- the ones in columns $i, j$ and $k$ are replaced by $\pi_{i}, \pi_{j}$ and $\pi_{k}$ respectively; in other words, we replace the submatrix corresponding to $M(p)$ with the pattern matrix $M^{\prime}(p)$,

- the zeros in columns $i, j$ and $k$ that are also in row $\pi_{i}, \pi_{j}$ or $\pi_{k}$ are replaced by plus signs,

- the trivial blocks in columns $i, j$ and $k$ are replaced by vertical bars, 


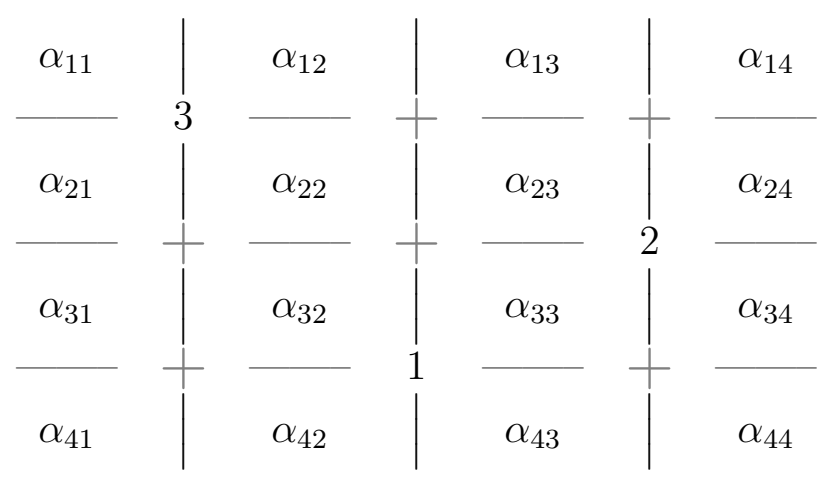

Figure 1: The lattice matrix $L_{312}(\pi)$

- the trivial blocks in rows $\pi_{i}, \pi_{j}$ and $\pi_{k}$ are replaced by horizontal bars, and finally,

- the conventional matrix brackets are omitted.

Note that we may also alter the ones, zeros and $\alpha_{i j}$ blocks (where $i, j \in[n+1]$ ) differently based on which properties of the permutation we are trying to highlight. This figure is reminiscent of the lattice structure of gridded window panes, so we call it the p-lattice matrix of $\pi$, or simply a lattice matrix, and denote it by $L_{p}(\pi)$.

In general, we may use the following definition:

Definition 24. Consider a permutation $\pi$ on $n$ letters and choose $m$ indices $1 \leqslant i_{1}<$ $i_{2}<\cdots<i_{m} \leqslant n$. Write $I=\left(i_{1}, i_{2}, \ldots, i_{m}\right)$ and let $p:=\operatorname{red}\left(\pi_{i_{1}} \pi_{i_{2}} \cdots \pi_{i_{m}}\right)$. We proceed to define the lattice matrix $L_{p}(\pi)$. Put $i_{0}=0$ and $i_{m+1}=n+1$. We use the values $i_{1}, i_{2}, \ldots, i_{m}$ to partition the column indices into subintervals and the values $\pi_{i_{1}}, \pi_{i_{2}}, \ldots, \pi_{i_{m}}$ to partition the row indices into subintervals.

A block in $L_{p}(\pi)$ is a (possibly trivial) continguous block submatrix of the permutation matrix $M(\pi)$ with its column and row indices each given by a relevant subinterval defined above. To make this more explicit, we note that $\pi\left(i_{p^{-1}(1)}\right)<\pi\left(i_{p^{-1}(2)}\right)<\cdots<\pi\left(i_{p^{-1}(m)}\right)$. Write $j_{k}:=\pi\left(i_{p^{-1}(k)}\right)$. Put $j_{0}=0$ and $j_{m+1}=n+1$. Then $M_{S \times T}$ where $S=\left[i_{s-1}+1, i_{s}-1\right]$ and $T=\left[j_{t-1}+1, j_{t}-1\right]$ is a block for all $s$ and $t$ in $[m+1]$. We label the block $M_{S \times T}$ as $\alpha_{s, t}$. Note that $\alpha_{s, t}$ is void if either $i_{s}=i_{s-1}$ or $j_{t}=j_{t-1}+1$. We may write $\alpha_{s, t}$ as $\alpha_{s t}$ if no confusion would arise. Note that $M_{i_{k}, \pi\left(i_{k}\right)}, M_{i_{k} \times T}$ and $M_{S \times j_{k}}$ may also be referred to as blocks for any $k \in[m]$ and subintervals $S$ and $T$ defined as above.

When depicting $L_{p}(\pi)$, we replace the zero entries in column $i_{k}$ by a vertical line and the zero entries in row $j_{k}$ by a horizontal line for every $k \in[m+1]$. We also omit the traditional matrix brackets around the entire matrix $L_{p}(\pi)$.

The following proposition is easy to prove:

Proposition 25. The matrix $L_{p}(\pi)$ defined above has the following properties:

1. $\left(\alpha_{s, t}\right)_{a, b}=M_{j_{s-1}+a, i_{t-1}+b}$ for $a \in\left[j_{s}-j_{s-1}-1\right]$ and $b \in\left[i_{t}-i_{t-1}-1\right]$ 
2. Each horizontal (respectively, vertical) bar is either void, or is a single row (respectively, column) of zeroes.

3. All block matrices in the same row (respectively, column) of the lattice matrix have the same number of rows (respectively, columns).

4. Any square block submatrix of the lattice matrix has the same total number of rows as columns.

We would like to describe the relationships between points in different blocks in a lattice matrix precisely. The following definitions provide an intuitive way to do so.

Definition 26. Let $p$ and $\pi$ be permutations of length $m$ and $n$ respectively where $m \leqslant n$ and $\pi$ contains $p$. Let $L_{p}(\pi)$ be the $p$-lattice matrix of $\pi$ with its blocks denoted by $\alpha_{i j}$ for $i, j \in[m+1]$.

(a) For the points in $L_{p}(\pi)$ that correspond to the pattern $p$, we define a point being adjacent to a block in a natural way. For example, in Figure 1, the point labelled 1 is adjacent to the blocks $\alpha_{32}, \alpha_{33}, \alpha_{42}$ and $\alpha_{43}$ while the point labelled 2 is adjacent to the blocks $\alpha_{23}, \alpha_{24}, \alpha_{33}$ and $\alpha_{34}$. We note that every point is adjacent to exactly four blocks.

(b) We use the four cardinal directions, north, south, east and west to to indicate where one point lies in relation to another in the visual representation of the permutation matrix $M(\pi)$. Explicitly, a point is north (respectively, south) of another point if and only if the row index in $M(\pi)$ of the former point is smaller than that of the latter, and is west (respectively, east) of another point if and only if the column index in $M(\pi)$ of the former point is smaller than that of the latter.

(c) For $i_{1}, i_{2} \in[m+1]$, we say that $\alpha_{i_{1} j}$ is to the left (respectively, to the right) of $\alpha_{i_{2} j}$ if and only if all the points in $\alpha_{i_{1} j}$ are west (respectively, east) of all the points in $\alpha_{i_{2} j}$.

(d) For $j_{1}, j_{2} \in[m+1]$, we say that $\alpha_{i j_{1}}$ is superior (respectively, inferior to $\alpha_{i j_{2}}$ if and only if all nontrivial columns of $\alpha_{i j_{2}}$ are north (respectively, south) of all nontrivial columns of $\alpha_{i j_{2}}$.

(e) We say that $\alpha_{i j}$ is leftmost (respectively, rightmost) if and only if the first (respectively, last) column of $\alpha_{i j}$ is nontrivial.

(f) We say that $\alpha_{i j}$ is topmost (respectively, bottommost) if and only if the first (respectively, last) row of $\alpha_{i j}$ is nontrivial.

\section{Permutations avoiding the $\operatorname{POP} \lambda$}

Gao and Kitaev [5] observed that the POP $\lambda$ of size 4 where $1>2$ and $1>4$ (illustrated in Figure 2) and the set of patterns

$$
\mathcal{P}=\{2413,2431,4213,3412,3421,4231,4321,4312\}
$$


are Wilf-equivalent. The proof in [5] was done by constructing a recurrence involving three unknowns which led them to derive the generating function corresponding to the OEIS sequence A111281. In this section, we prove that $\lambda$ and $\mathcal{P}$ are Wilf-equivalent by deriving the recursive equation in A111281 and constructing an explicit bijection from $A v(\lambda)$ to $A v(\mathcal{P})$.

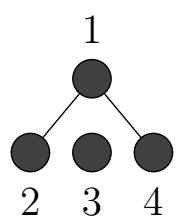

Figure 2: The POP $\lambda$

First, we show that both avoidance sets have only finitely many simple permutations. Using this fact, we analyse the possible inflations of these simple permutations in each avoidance set and derive a method to construct each set recursively. A recursively-defined bijection on the two sets follows immediately from this analysis.

In this section, we use the symbol $I_{k}$ to denote the identity permutation $123 \cdots k$ for $k \geqslant 1$.

\subsection{Structure of permutations avoiding the POP $\lambda$}

Lemma 27. The only simple permutations that avoid $\lambda$ are 12, 21 and 2413.

Proof. It is clear that 2413 and all simple permutations of length 3 or less avoid $\lambda$. Consider the permutation matrix of a simple permutation $\pi$ with length at least 4 . It must contain the pattern 312 by Corollary 13 , say $1 \leqslant i<j<k \leqslant n$ where $\operatorname{red}\left(\pi_{i} \pi_{j} \pi_{k}\right)=312$. We can then consider the lattice matrix $L_{312}(\pi)$ which is illustrated in Figure 1 . It suffices to prove that $\alpha_{31}$ has weight 1 , while the other blocks are trivial.

Upon inspection, it is clear that if any of $\alpha_{11}, \alpha_{13}$ or $\alpha_{i j}$, where $i, j \in[2,4]$, were not trivial, then the permutation would contain $\lambda$. For the reader's convenience, we reproduce the figure with those $\alpha_{i j}$ 's omitted in Figure 3. We now proceed to show that the remaining blocks, except for $\alpha_{31}$, are trivial:

(a) Suppose $\alpha_{41}$ is not trivial. It cannot be to the left of $\alpha_{21}$ and $\alpha_{31}$ as this would mean that the permutation is sum decomposable. So $\alpha_{21}$ or $\alpha_{31}$ contains a point west of a point in $\alpha_{41}$. However, these two points together with the points labelled 3 and 1 yields the POP $\lambda$. So $\alpha_{41}$ must be trivial.

(b) Suppose $\alpha_{21}$ is not trivial. Since it is adjacent to the block labelled 3, it cannot be to the right of $\alpha_{31}$ since $\pi$ is simple. So $\alpha_{31}$ contains a point east of a point of $\alpha_{21}$. However, these two points together with the points labelled 3 and 1 yields the POP $\lambda$. So $\alpha_{21}$ must be trivial.

(c) Suppose $\alpha_{14}$ is not trivial. We know that $\alpha_{12}$ must be nontrivial and superior to $\alpha_{14}$ since the permutation would be sum decomposable otherwise. Consider a point in 


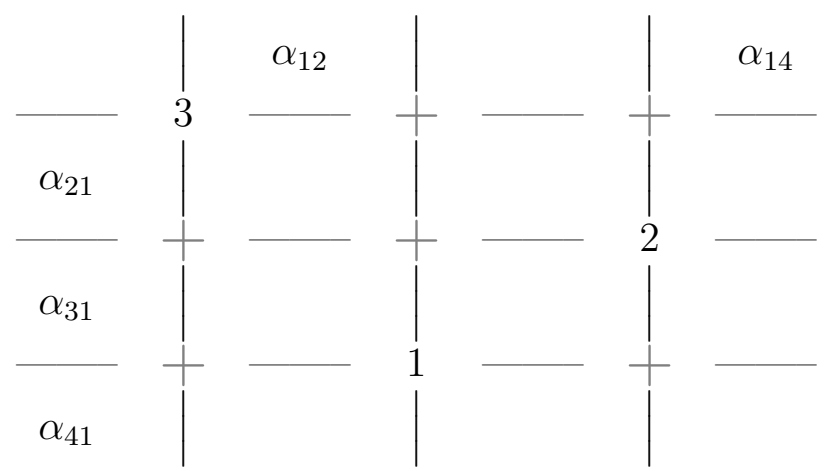

Figure 3: The lattice matrix $L_{312}(\pi)$, with some blocks omitted. The omitted blocks must be trivial for $\pi$ to avoid $\lambda$.

$\alpha_{12}$ north to a point in $\alpha_{14}$, together with the points labelled 1 and 2 . These points yield the $\operatorname{POP} \lambda$. So $\alpha_{14}$ must be trivial.

(d) Finally, observe that $\alpha_{12}$ is adjacent to the block labelled 3. Since the blocks in the same row or column as $\alpha_{12}$ are trivial, $\alpha_{12}$ must also be trivial by simpleness.

Since all the blocks in the same row or column as $\alpha_{31}$ are trivial, it can have weight at most 1 by simpleness. So 2413 is the only simple permutation of length at least 4 avoiding $\lambda$.

Lemma 28. For $n \geqslant 4$, there are $n$ skew sum decomposable permutations in $A v_{n}(\lambda)$, namely $21\left[I_{n-1}, 12\right], 21\left[I_{n-2}, 21\right]$ and $2431\left[I_{\ell}, I_{n-\ell-2}, 1,1\right]$ where $\ell \in[2, n-1]$.

Proof. Let $\pi=21[\alpha, \beta]$ be an $n$-permutation avoiding $\lambda$. It is not hard to see that if $|\beta| \geqslant 3$, then $\pi$ contains $\lambda$. So $|\beta|=1$ or 2 :

1) Suppose $|\beta|=2$. If $\alpha$ contains a descent, then the elements that make up the descent, together with $\beta$ make up $\lambda$. So $\alpha$ must be an increasing sequence, and indeed both $21\left[I_{n-1}, 12\right]$ and $21\left[I_{n-2}, 21\right]$ avoid $\lambda$.

2) Suppose $|\beta|=1$. Then $\pi$ avoids $\lambda$ if and only if $\alpha$ avoids the POP of size 3 where $1>2$. This is exactly when the first $n-2$ elements of $\alpha$ are increasing. Since $\alpha$ is assumed to be skew sum indecomposable (for uniqueness), the last element of $\alpha$ cannot be 1 . Therefore there are $n-2$ choices for the last element of $\alpha$, and only one way to order the initial elements. Indeed, for all $2 \leqslant \ell \leqslant n-1$, the following permutation avoids $\lambda$ :

$$
\begin{aligned}
\pi & =21[12 \cdots \ell(\ell+2) \cdots(n-2)(\ell+1), 1] \\
& =21\left[132\left[I_{\ell}, I_{n-\ell-2}, 1\right], 1\right] \\
& =2431\left[I_{\ell}, I_{n-\ell-2}, 1,1\right]
\end{aligned}
$$

Therefore, there are $(n-2)+2=n$ skew sum decomposable $n$-permutations avoiding $\lambda$ in total.

Lemma 29. For $n \geqslant 4$, there are $n-3$ permutations that are inflations of 2413 avoiding $\lambda$ that are of length $n$. Specifically, they are of the form $2413\left[I_{\ell}, I_{n-\ell-2}, 1,1\right]$ for $\ell \in[n-3]$. 
Proof. Let $\pi:=2413[\alpha, \beta, \gamma, \delta]$ be an $n$-permutation avoiding $\lambda$. We will show that $|\gamma|=|\delta|=1$, while $\alpha$ and $\beta$ are increasing sequences but can be of variable length.

Suppose $|\gamma| \geqslant 2$ or $|\delta| \geqslant 2$. Then $\pi$ contains $\lambda$ (consider one point from $\beta$ ) and three points total from $\gamma$ and $\delta$. Now suppose that $\alpha$ or $\beta$ contains a descent. Then the two elements that make up the descent, together with one element from $\gamma$ and one element from $\delta$ make $\lambda$. Therefore, $|\gamma|=|\delta|=1$ and $\alpha$ and $\beta$ are increasing.

Finally, it is not hard to see that for all $\ell \in[n-3]$, the permutation $2413\left[I_{\ell}, I_{n-\ell-2}, 1,1\right]$ avoids $\lambda$. So there are $n-3$ inflations of 2413 avoiding $\lambda$.

Theorem 30. For all $n \geqslant 4,\left|A v_{n}(\lambda)\right|=2 n-3+\sum_{i=1}^{n-1}(2 i-3)\left|A v_{n-i}(\lambda)\right|$.

Proof. Lemmas 27, 28 and 29 together imply that there are $2 n-3$ sum indecomposable permutations in $A v_{n}(\lambda)$. Moreover, a sum decomposable permutation $12[\alpha, \beta]$ avoids $\lambda$ if and only if $\alpha$ and $\beta$ both avoid $\lambda$. Therefore, there are $\sum_{i=1}^{n-1}(2 i-3)\left|A v_{n-i}(\lambda)\right|$ sum decomposable permutations of the form $12[\alpha, \beta]$ in $A v_{n}(\lambda)$, where $\alpha$ is sum indecomposable. Thus we get the recursive formula for $A v_{n}(\lambda)$.

\section{$2.2 \quad$ Structure of permutations avoiding $\mathcal{P}$}

Next, we present the $n$-permutations of $A v(\mathcal{P})$ explicitly. Recall that

$$
\mathcal{P}=\{2413,2431,4213,3412,3421,4231,4321,4312\} .
$$

Lemma 31. The only simple permutations that avoid $\mathcal{P}$ are 12, 21, 3142 and 41352.

Proof. It is clear that 3142, 41352 and all simple permutations of length 3 or less avoid $\mathcal{P}$. Consider a simple permutation $\pi$ of length at least 4 avoiding $\mathcal{P}$. Since $\mathcal{P}$ contains 2413, $\pi$ avoids 2413 and must contain 3142, since otherwise it would be a separable permutation and not simple by Theorem 11 .

We present its lattice matrix $L_{3142}(\pi)$ in Figure 4, with some alterations explained in the caption. It suffices to show that $\alpha_{33}$ can have weight at most 1 , while the remaining $\alpha_{i j}$ are trivial:

We proceed to show that $\alpha_{i j}$ must be trivial for all $i, j \in[5]$, except for $i=j=3$.

(a) Suppose $\alpha_{52}$ were nontrivial. Since it is adjacent to the point 1, the block $\alpha_{51}$ cannot be trivial and must be superior to $\alpha_{52}$ by simpleness. However, this would mean the inclusion of the pattern 2413 (consider any submatrix containing $\alpha_{51} 3 \alpha_{52} 1$ ).

(b) Since $\alpha_{5 j}$ are trivial for all $j \in[2,5]$, the block $\alpha_{51}$ must be trivial as well, for otherwise $\pi$ would be sum decomposable.

(c) Suppose $\alpha_{14}$ were nontrivial. Since it is adjacent to the point 4 , the block $\alpha_{15}$ cannot be trivial and must be inferior to $\alpha_{14}$ by simpleness. However, this would mean the inclusion of the pattern 2413 (consider any submatrix containing $4 \alpha_{14} 2 \alpha_{15}$ ). 


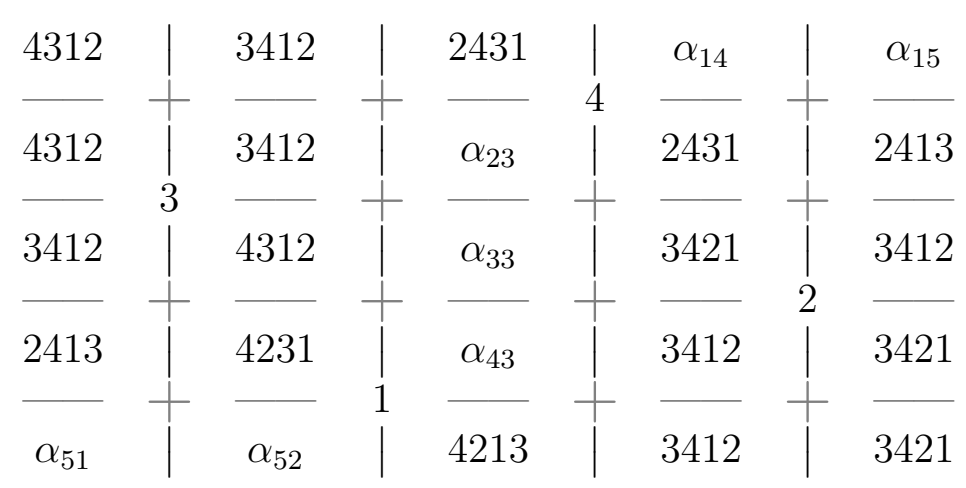

Figure 4: The lattice matrix $L_{3142}(\pi)$ with some $\alpha_{i j}$ replaced by a pattern in $\mathcal{P}$ that $\pi$ would contain if that $\alpha_{i j}$ were nontrivial, for $i, j \in[5]$. For example, if $\alpha_{12}$ were nontrivial, then $\pi$ would contain 3412 .

(d) Suppose $\alpha_{23}$ were nontrivial. Since it is adjacent to the point 4, it cannot be rightmost by simpleness. However, this would mean the inclusion of the pattern 3412 (consider any submatrix containing $3 \alpha_{23} \alpha_{33} 2$ or $3 \alpha_{23} \alpha_{43} 2$ ).

(e) Suppose $\alpha_{43}$ were nontrivial. Since it is adjacent to the point 1, it cannot be leftmost by simpleness. Then $\alpha_{33}$ must be nontrivial and lie to the left of $\alpha_{43}$. However, this would mean the inclusion of the pattern 4312 (consider any submatrix containing $3 \alpha_{33} \alpha_{43} 2$ ).

Since all the blocks in the same row or column as $\alpha_{33}$ are trivial, it can have weight at most 1 by simpleness. Moreover, it cannot be trivial since all the other $\alpha_{i j}$ s are trivial and 312 is not simple. We have thus eliminated the possibility of there being a simple permutation of length at least 5 avoiding $\mathcal{P}$ that is not 41352, so our list is exhaustive.

Lemma 32. There are 4 skew sum decomposable n-permutations in $A v(\mathcal{P})$. Namely, they are $21\left[1, I_{n-1}\right], 312\left[1, I_{n-3}, 21\right], 21\left[I_{n-1}, 1\right]$ and $231\left[21, I_{n-3}, 1\right]$.

Proof. Let $\pi=21[\alpha, \beta]$ be a permutation avoiding $\mathcal{P}$. We have 3 cases:

1) If $|\alpha|,|\beta| \geqslant 2$, then $\pi$ contains $3412,3421,4312$ or 4321.

2) Suppose $|\alpha|=1$ and $|\beta| \geqslant 2$. Then $\pi$ avoids $\mathcal{P}$ if and only if $\beta$ avoids $213,231,321$ and 312 .

That is, $\pi$ avoids $\mathcal{P}$ if and only if all but the last two terms of $\beta$ are strictly increasing. There are only two such permutations, namely $21\left[1, I_{n-1}\right]$ and $312\left[1, I_{n-3}, 21\right]$.

3) Suppose $|\beta|=1$ and $|\alpha| \geqslant 2$. Then $\pi$ avoids $\mathcal{P}$ if and only if $\alpha$ avoids

$$
\operatorname{red}(243)=132, \quad \operatorname{red}(342)=231, \quad \operatorname{red}(423)=312 \quad \text { and } \quad \operatorname{red}(432)=321 .
$$

That is, if and only if all but the first two terms of $\alpha$ are strictly increasing.

There are only two such permutations, namely $21\left[I_{n-1}, 1\right]$ and $231\left[21, I_{n-3}, 1\right]$.

Lemma 33. There are $n-3$ inflations of 3142 of length $n$ avoiding $\mathcal{P}$. Specifically, they are of the form $3142\left[1, I_{\ell}, I_{n-\ell-2}, 1\right]$ where $\ell \in[n-3]$. 
Proof. Let $\pi=3142[\alpha, \beta, \gamma, \delta]$ be a permutation avoiding $\mathcal{P}$. We will show that $|\alpha|=$ $|\delta|=1$, while $\beta$ and $\gamma$ are increasing sequences of variable length.

1. If $\alpha$ contains an ascent, then $\pi$ contains 3412 (consider $312[\alpha, \beta, \delta]$ ).

On the other hand, if $\alpha$ contains an descent, then $\pi$ contains 4312 (consider $312[\alpha, \beta, \delta])$.

2. If $\delta$ contains an ascent, then $\pi$ contains 3412 (consider 342[]$\alpha, \gamma, \delta]$ ).

On the other hand, if $\delta$ contains an descent, then $\pi$ contains 3421 (consider the subpermutation $342[\alpha, \gamma, \delta])$.

3. If $\beta$ contains an descent, then $\pi$ contains 4213 (consider $314[\alpha, \beta, \delta]$ ).

If $\gamma$ contains an descent, then $\pi$ contains 2431 (consider $342[\alpha, \gamma, \delta]$ ). Therefore $\beta$ and $\gamma$ are increasing.

Finally, it is not hard to see that for all $\ell \in[n-3]$, the permutation $3142\left[1, I_{\ell}, I_{n-\ell-2}, 1\right]$ avoids $\lambda$. Therefore, there are $n-3$ inflations of 3142 in $A v_{n}(\mathcal{P})$.

Lemma 34. There are $n-4$ inflations of 41352 of length $n$ avoiding $\mathcal{P}$. Specifically, they are of the form $41352\left[1, I_{\ell}, 1, I_{n-\ell-3}, 1\right]$ where $\ell \in[n-4]$.

Proof. Let $41352[\alpha, \beta, \gamma, \delta, \zeta]$ be a permutation avoiding $\mathcal{P}$. We will show that $|\alpha|=|\gamma|=$ $|\zeta|=1$, while $\beta$ and $\delta$ are both increasing sequences of variable length.

1. If $\alpha$ contains an ascent, then $\pi$ contains 3412 (consider $413[\alpha, \beta, \gamma]$ ).

If $\alpha$ contains an descent, then $\pi$ contains 4312 (consider $413[\alpha \beta, \gamma]$ ).

Therefore $|\alpha|=1$.

2. If $\gamma$ contains an ascent, then $\pi$ contains 4231 (consider $432[\alpha, \gamma, \zeta]$ ).

If $\gamma$ contains an descent, then $\pi$ contains 4231 (consider $432[\alpha, \gamma, \zeta]$ ).

Therefore $|\gamma|=1$.

3. If $\zeta$ contains an ascent, then $\pi$ contains 4312 (consider $432[\alpha, \gamma, \zeta]$ ).

If $\zeta$ contains an descent, then $\pi$ contains 4321 (consider $432[\alpha, \gamma, \zeta]$ ).

Therefore $|\zeta|=1$.

4. If $\beta$ contains an descent, then $\pi$ contains 4213 (consider $412[\alpha, \beta, \zeta]$ ).

Therefore $\beta$ is increasing.

5. If $\delta$ contains an descent, then $\pi$ contains 2431 (consider $452[\alpha, \delta, \zeta]$ ).

Therefore $\delta$ is increasing.

Finally, it is not hard to see that for all $\ell \in[n-4]$, the permutation $41352\left[1, I_{\ell}, 1, I_{n-\ell-3}, 1\right]$ avoids $\mathcal{P}$. Therefore, there are $n-4$ inflations of 41352 in $A v_{n}(\mathcal{P})$.

Theorem 35. For all $n \geqslant 4,\left|A v_{n}(\mathcal{P})\right|=2 n-3+\sum_{i=1}^{n-1}(2 i-3)\left|A v_{n-i}(\mathcal{P})\right|$.

Proof. From Lemma 31, 32, 33 and 34, we can easily see that there are $2 n-3$ sum indecomposable $n$-permutations in $A v(\mathcal{P})$. Moreover, a sum decomposable $n$-permutation $12[\alpha, \beta]$ avoids $\mathcal{P}$ if and only if $\alpha$ and $\beta$ both avoid $\mathcal{P}$, so there are $\sum_{i=1}^{n-1}(2 i-3)\left|A v_{n-i}(\mathcal{P})\right|$ permutations of the form $12[\alpha, \beta]$ in $A v_{n}(\mathcal{P})$ where $\alpha$ is sum indecomposable. 


\subsection{The bijection}

Having thoroughly analysed the structure of the two avoidance sets, we are ready to construct the bijection explicitly:

Theorem 36. Define $g$ to be a function that maps

$$
\begin{aligned}
1 & \mapsto 1 \\
321 & \mapsto 321 \\
312 & \mapsto 231 \\
21\left[I_{k}, 1\right] & \mapsto 21\left[1, I_{k}\right] \\
231\left[I_{k}, 21,1\right] & \mapsto 312\left[1, I_{k}, 21\right] \\
21\left[I_{k+1}, 21\right] & \mapsto 231\left[21, I_{k}, 1\right] \\
2431\left[I_{k}, I_{j+1}, 1,1\right] & \mapsto 41352\left[1, I_{k}, 1, I_{j}, 1\right] \\
2413\left[I_{k}, I_{j}, 1,1\right] & \mapsto 3142\left[1, I_{k}, I_{j}, 1\right]
\end{aligned}
$$

for all $k, j \geqslant 1$. Define $f: A v(\lambda) \rightarrow A v(\mathcal{P})$ by

$$
f(\pi)= \begin{cases}g(\pi) & \text { if } \pi \text { is sum indecomposable } \\ 12[g(\alpha), f(\beta)] & \text { if } \pi=12[\alpha, \beta], \text { and } \alpha \text { is a sum indecomposable. }\end{cases}
$$

Then $f$ is a bijection. In fact, $f$ restricted to $A v_{n}(\lambda)$ is a bijection onto $A v_{n}(\mathcal{P})$.

Proof. From the lemmas in the previous two sections, it is clear that $g$ maps the sum indecomposable permutations in $A v_{n}(\lambda)$ to the sum indecomposable permutations in $A v_{n}(\mathcal{P})$. Moreover $f$ maps permutations in $A v_{n}(\lambda)$ into $A v_{n}(\mathcal{P})$ by the proofs of Theorem 30 and Theorem 35, and it is clear that $f$ is an injection. Since $\left|A v_{n}(\lambda)\right|=\left|A v_{n}(\lambda)\right|, f$ restricted to $A v_{n}(\lambda)$ is a bijection onto $A v_{n}(\mathcal{P})$ for all $n \geqslant 1$. Thus $f$ is a bijection from $A v(\lambda)$ to $A v(\mathcal{P})$.

\section{$3 Q_{k}$ and two connections to classical combinatorial objects}

Definition 37. Let $Q_{k}$ be the POP with $k$ elements where $1>t$ for $t \in[2, n]$. The Hasse diagram of $Q_{k}$ is the following:

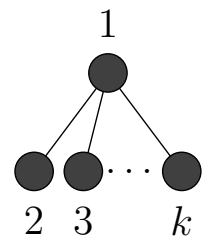

Figure 5: The POP $Q_{k}$

Gao and Kitaev [5] enumerated the avoidance set of $Q_{k}$ in Theorem 2 of their paper and observed that the sequences $\left|A v_{n}\left(Q_{4}\right)\right|_{n \geqslant 1}$ and $\left|A v_{n}\left(Q_{5}\right)\right|_{n \geqslant 1}$ are listed in the OEIS database as A025192 and A084509, respectively. These sequences enumerate the 2-ary shrub forests of $n$ heaps avoiding the patterns 231, 312 and 321, and the ground state 
3-ball juggling sequence of period $n$, respectively. These objects will be defined in the following sections.

We show that there are intimate connections between the relevant sets by constructing explicit bijections. In fact, we construct an explicit bijection from $A v_{n}\left(Q_{k}\right)$ to ground state $(k-2)$-ball juggling sequence of period $n$ which yields a bijection between the original two sets as a special case. The study that led us to this bijection also produced a new way of enumerating the avoidance sets of $Q_{k}$ using the concept of matrix permanents.

\subsection{Enumeration of $A v_{n}\left(Q_{k}\right)$}

We will enumerate $A v_{n}\left(Q_{k}\right)$ for $k, n \geqslant 1$ using the concept of matrix permanents. Recall that the permanent is defined on square matrices and is similar to the definition of the determinant of a matrix, where the signs of the summands are all positive instead of alternating. We restate Gao and Kitaev's proof first as a reference for comparison.

Theorem 38 (Gao and Kitaev (2019) [5]). For $n \geqslant k,\left|A v_{n}\left(Q_{k}\right)\right|=(k-1) ! \times(k-1)^{n-k+1}$.

Proof. We proceed by induction. It is clear that all $(k-1)$-permutations avoid $Q_{k}$, so $\left|A v_{k-1}\left(Q_{k}\right)\right|$ is $(k-1)$ !. For $n \geqslant k, \pi$ is an $n$-permutation avoiding $Q_{k}$ if and only if $n \in$ $\pi_{[n-k+2, n]}$ and the $(n-1)$-permutation obtained from removing $n$ from the permutation $\pi$ avoids $Q_{k}$. So $\left|A v_{n}\left(Q_{k}\right)\right|=(k-1) \times\left|A v_{n-1}\left(Q_{k}\right)\right|$. The desired formula can then be easily obtained from this recursion.

Definition 39. The permanent of an $n \times n$ matrix $A_{n}=\left(a_{i j}\right)_{i, j \in[n]}$ is

$$
\operatorname{Perm}\left(A_{n}\right):=\sum_{\pi \in S_{n}} \prod_{i=1}^{n} a_{i \pi_{i}} .
$$

The following proposition states a well-known property of permanents. Its proof is similar to the proof for an analogous statement for determinants and is therefore omitted.

Proposition 40 (folklore). The permanent of a matrix is invariant under arbitrary permutations of the rows and/or columns, as well as transposition. That is, for all $n \times n$ matrices $M$ and $n$-permutation matrices $P$ and $Q$,

$$
\operatorname{Perm}\left(M^{T}\right)=\operatorname{Perm}(M)=\operatorname{Perm}(P M Q) .
$$

Observe that the permanent of the $n \times n$ matrix of ones is equal to $n$ ! for all positive $n$, and recall that there are $n$ ! permutations in $S_{n}$. One may ask whether there is a matrix associated with subsets of $S_{n}$, such that the problem of enumerating those subsets can be converted into computing a certain value of a matrix. This is in fact possible for certain subsets of $S_{n}$, as we shall see.

Lemma 41 (Percus (1971) [7]). Let $A_{n}$ be a binary $n \times n$ matrix for some $n \geqslant 1$. Define $R_{n}$ to be the set of $n$-permutations such that for all $i \in[n]$ and $\pi \in R_{n}$, the $i \pi_{i}$ th element of $A_{n}$ is 1. Then $\left|R_{n}\right|$ is the permanent of $A_{n}^{K}$. 
Proof. Observe that

$$
\#\left\{\pi \in S_{n} \mid a_{i \pi_{i}}=1 \text { for all } i \in[1, n]\right\}=\sum_{\pi \in S_{n}} a_{1 \pi_{1}} a_{2 \pi_{2}} \cdots a_{n \pi_{n}}=\sum_{\pi \in S_{n}} \prod_{i=1}^{n} a_{i \pi_{i}}=\operatorname{Perm}(A) .
$$

Theorem 42. Let $n \geqslant 1$ and $A_{n, k}$ be a binary $n \times n$ matrix where $a_{i j}=1$ if and only if $i \geqslant j-k+2$. Then $A v_{n}\left(Q_{k}\right)$ is the set of $n$-permutations such that for all $i \in[n]$ and $\pi \in A v_{n}\left(Q_{k}\right)$, the $i \pi_{i}$ th element of $A_{n, k}$ is 1 .

Proof. Observe that an $n$-permutation $\pi$ avoids $Q_{k}$ if and only if $t \in \pi_{[t-k+2, n]}$ for all $t \in[k, n]$. That is, $\pi_{i}=t \Longleftrightarrow i \in[t-k+2, n] \Longleftrightarrow i \geqslant t-k+2$ for all $i \in[n]$.

Since the permanent of $A_{n}, k$ is exactly $(k-1) !(k-1)^{n-k+1}$ for all $n \geqslant k$, the theorem above proves the enumeration of the avoidance set of $Q_{k}$ for all $n$.

\section{$3.2 \quad$ Juggling sequences}

Suppose we have $b$ balls and a binary vector $\sigma=\left(\sigma_{1}, \sigma_{2}, \ldots, \sigma_{n}\right) \in\{0,1\}^{n}$ for some integer $n \geqslant b$. Given a reference time, we can throw one ball at the $i$ th second such that it lands in our hand again after exactly $t_{i}$ seconds (that is, $i+t_{i}$ seconds after the reference time) where $t_{i}$ is a positive integer for all $i \in[n]$, if $\sigma_{i}=1$. Otherwise, we put $t_{i}:=0$. We say that the $n$-tuple of non-negative integers $T=\left(t_{1}, t_{2}, \ldots, t_{n}\right)$ is a juggling sequence of period $n$ and state $\sigma$ if and only if no two balls land in our hand at the same time, and our sequence of throws is infinitely repeatable. That is, the following two conditions hold:

- $i+t_{i} \equiv j+t_{j}(\bmod n)$ if and only if $i=j$ for all $i, j \in[n]$,

- $\sigma_{i}=1$ if and only if there is some $j \in[n]$ such that $i \equiv j+t_{j}(\bmod n)$.

We say that $\sigma$ is a ground state if and only if $\sigma_{i}=1$ if $i \in[b]$ and $\sigma_{i}=0$ otherwise. We refer the reader to [3] and [4] for diagrams and further analysis on juggling sequences.

Gao and Kitaev [5] observed that the avoidance set $A v_{n}\left(Q_{5}\right)$ and the number of ground-state 3-ball juggling sequences of period $n$ are enumerated by the same OEIS sequence A084509. We will provide a natural bijection between a generalization of these two combinatorial objects, which is inspired by the proof of Theorem 1 of [4], restated below:

Theorem 43. The number of ground state juggling sequences of period $n$ using $b$ balls $(n \geqslant b)$ is

$$
J(n, b)= \begin{cases}(b+1)^{n-b} b ! & \text { if } n \geqslant b, \\ n ! & \text { otherwise. }\end{cases}
$$

Proof. Observe that $T=\left(t_{1}, t_{2}, \ldots, t_{n}\right)$ is a conforming juggling sequence if and only if every ball that is thrown lands exactly at some time $t$ seconds after the reference time where $t \in[n+1, n+b]$, and no two balls land on the same second. That is, $\left\{t_{i}+i \mid i \in[b]\right\}=[n+1, n+b]$. Since $t_{i}=0$ for $i \in[n] \backslash[b]$, the condition is equivalent 
to requiring $\left\{t_{i}+i-b \mid i \in[n]\right\}=[n]$. That is, the bijection $i \mapsto t_{i}+i-b$ defines a permutation on the set $[n]$, with the additional condition that $t_{i} \geqslant 0$ for all $i \in[n]$. Thus every conforming juggling sequence $T$ can be associated uniquely to a permutation $\pi$ where $\pi_{i}=t_{i}+i-b$. The number of such permutations is then exactly the permanent of the matrix $M$ where its $i j$ th entry is 1 if and only if $j-i+b \geqslant 0$, by Lemma 41 . Computing the permanent of $M$ yields the formula assigned to $J(n, b)$.

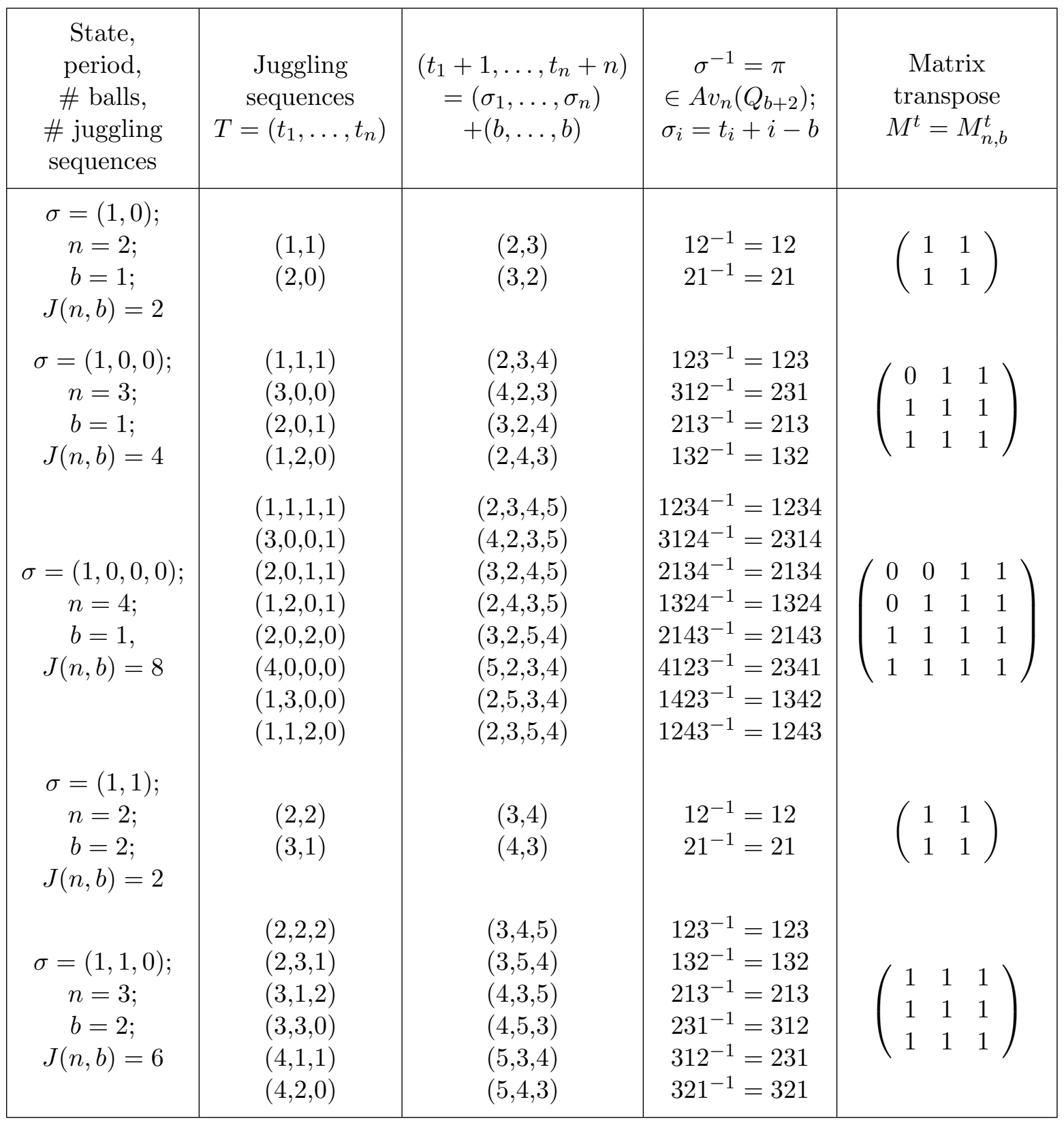

Table 2: Sample values for juggling sequences and their images under $\theta$ for small $n$ and $b$. Note that we are using the permutation matrix notation system where rows are numbered from bottom to top in increasing order.

The desired bijection can then be easily obtained by realizing that the permutations mentioned in the proof of Theorem 43 are exactly the permutation inverses of those 
avoiding $Q_{b+2}$, as is carefully fleshed out in the following theorem:

Theorem 44. Let $\theta$ be a function from the set of ground state juggling sequences of period $n$ using $b$ balls to $A v_{n}\left(Q_{b+2}\right)$ given by $\theta\left(\left(t_{1}, t_{2}, \ldots, t_{n}\right)\right)=\pi$ where $\pi_{t_{i}+i-b}=i$ for all $i \in[n]$. Then $\theta$ is a bijection.

Proof. Since $t_{i}$ is nonnegative for all $i \in[n]$, we have

$$
\pi_{t_{i}+i-b}=i \Longleftrightarrow \pi_{i}=i+b-t_{i} \Longleftrightarrow i \geqslant \pi_{i}-b=\pi_{i}-i-(b+2)+2 .
$$

Therefore, for a given $n, b$, the matrix $M$ defined in Chung and Graham's paper is exactly the transpose of the matrix associated with $A v_{n}\left(Q_{b+2}\right)$ in Theorem 42. So the codomain of $\theta$ is indeed $A v_{n}\left(Q_{b+2}\right)$. By Theorem 38, the number of ground state $b$-ball juggling sequences of period $n$ is equal to the size of $A v_{n}\left(Q_{b+2}\right)$ since

$$
A v_{n}\left(Q_{b+2}\right)= \begin{cases}(b+1) ! \times(b+1)^{n-b-1}=(b+1)^{n-b} b ! & \text { if } n \geqslant b+2, \\ n ! & \text { otherwise. }\end{cases}
$$

Therefore, since $\theta$ is injective, it must also be bijective.

We refer the reader to Table 3.2 for sample values for small $n$ and $b$.

\subsection{Shrub forests of $n$ heaps}

Definition 45. Let $\mathcal{P}_{3 n}$ denote the set of permutations of length $3 n$ that avoid the patterns 231, 312 and 321 and satisfies $\pi_{3 i+1}<\pi_{3 i+2}$ and $\pi_{3 i+1}<\pi_{3 i+3}$ for all $i \in[n-1]$.

Remark 46. $\mathcal{P}_{3 n}$ is also known as the set of 2-ary shrub forests of $n$ heaps avoiding the patterns 231, 312 and 321.

Gao and Kitaev [5] observed that $A v_{n}\left(Q_{4}\right)$ and the $\mathcal{P}_{3 n}$ are enumerated by the same OEIS sequence A025192. We will show a natural bijection between these two sets for all $n \geqslant 1$.

Theorem 47. Let

$$
P_{3 n}=\left\{\begin{array}{ll}
\{123,132\} & \text { if } n=1, \\
\left\{1 \oplus \tau \oplus \pi_{[2,3 n-3]} \mid \tau \in\{123,132,213\} \text { and } \pi \in \mathcal{P}_{3 n-3}\right\} & \text { if } n \geqslant 2 .
\end{array} .\right.
$$

Then $P_{3 n}=\mathcal{P}_{3 n}$.

Proof. It is easy to see that $\mathcal{P}_{3}=\{123,132\}$. Let $\sigma:=1 \oplus \tau \oplus \pi_{[2,3 n-3]}$ for some $\tau \in\{123,132,213\}$ and $\pi \in \mathcal{P}_{3 n-3}$. It is clear that

$$
|\sigma|=\left|1 \oplus \tau \oplus \pi_{[2,3 n]}\right|=1+3+\left|\pi_{[2,3 n]}\right|=4+3 n-3-1=3 n,
$$

so $\sigma$ is a $3 n$-permutation. We know that $\pi \in \mathcal{P}_{3 n-3}$, so $\sigma_{3 i+1}<\sigma_{3 i+2}, \sigma_{3 i+3}$ for $i=$ $3,4, \ldots, n-1$. By the definition of the direct sum $\oplus$ we have $\sigma_{1}<\sigma_{[2,4]}<\sigma_{[5,3 n]}$, so $\sigma_{3 i+1}<\sigma_{3 i+2}$ and $\sigma_{3 i+1}<\sigma_{3 i+3}$ for $i=1$ and 2 as well. Since $\pi_{[2,3 n-3]}$ and $\tau=$ $S_{3} \backslash\{231,312,321\}$ both avoid 231, 312 and 321 , so do the factors $\sigma_{[1,4]}$ and $\sigma_{[5,3 n]}$. It is easy to see (by the definition of $\oplus$ ) that none of the forbidden patterns may span across 
$\sigma_{[1,4]}$ and $\sigma_{[5,3 n]}$. Therefore $\sigma \in \mathcal{P}_{3 n}$.

We claim that $\left|P_{3 n}\right|=2 \times 3^{n-1}$ for all $n \geqslant 1$. Indeed, $\left|P_{3}\right|=2=2 \times 3^{0}$. Suppose this is true for some $k \geqslant 2$. Since $\pi_{1}=1$ for all $\pi \in P_{3 k}$, we must have that $\pi_{[2,3 k]}$ is distinct for each $\pi \in P_{3 k}$. Therefore $\left|P_{3 k+3}\right|=3 \times\left|P_{3 k}\right|=2 \times 3^{n-1}$, and the claim is true for all $n$. Since $P_{3 n} \subseteq \mathcal{P}_{3 n}$ and $\left|P_{3 n}\right|=|\mathcal{P}|_{3 n}$, they must be equal for all $n \geqslant 1$.

Theorem 48. Let $\theta: A v_{n}\left(Q_{4}\right) \rightarrow \mathcal{P}_{3 n-3}$ be given by

$$
\begin{aligned}
& \theta(12)=123, \quad \theta(21)=132, \quad \text { and for }|\pi|=n \geqslant 3, \\
& \theta(\pi)= \begin{cases}1 \oplus 132 \oplus \theta\left(\pi_{[1, n-1]}\right)_{[2,3 n]}, & \text { if } \pi_{n}=n, \\
1 \oplus 123 \oplus \theta\left(\pi_{[1, n-2]} \pi_{n}\right)_{[2,3 n]}, & \text { if } \pi_{n-1}=n, \\
1 \oplus 213 \oplus \theta\left(\pi_{[1, n-3]} \pi_{[n-1, n]}\right)_{[2,3 n]} & \text { if } \pi_{n-2}=n .\end{cases}
\end{aligned}
$$

Then $\theta$ is a bijection.

Proof. Recall that $k$ can only be $\pi_{n}, \pi_{n-1}$ or $\pi_{n-2}$ by the proof of Theorem 42. So $\theta$ is defined on $A v_{n}\left(Q_{4}\right)$ for $n \geqslant 3$. Moreover, $\theta\left(A v_{n}\left(Q_{4}\right)\right) \subseteq S_{3 n-3}$ for all $n \geqslant 2$. The base case is: $|\theta(12)|=|\theta(21)|=3$. Suppose $\theta\left(A v_{k-1}\left(Q_{4}\right)\right) \subseteq S_{3 k-6}$ for some $k \geqslant 3$. Then if $\pi \in A v_{k}\left(Q_{4}\right)$, we have

$$
|\theta(\pi)|=1+3+(3(k-1)-2+1)=3 k=3(k+1)-3 .
$$

So $\theta$ maps into $S_{3 n-3}$ for all $n$. Obviously $\theta\left(A v_{2}\left(Q_{4}\right)\right)=\mathcal{P}_{3}$. Suppose $\theta\left(A v_{k-1}\left(Q_{4}\right)\right) \subseteq$ $\mathcal{P}_{3 k-6}$ for some $k \geqslant 2$. Then by Theorem $47, \theta$ indeed maps $A v_{k}\left(Q_{4}\right)$ into $\mathcal{P}_{3 k-3}$. So $\theta\left(A v_{n}\left(Q_{4}\right)\right) \subseteq \mathcal{P}_{3 n-3}$ for all $n \geqslant 2$ by induction.

Finally, it is clear from the definition of $\theta$ that it is injective. By Theorem 38, $\left|A v_{n}\left(Q_{4}\right)\right|=3 ! \times 3^{n-3}=2 \times 3^{n-2}$ for $n \geqslant k$. Since $\left|A v_{n}\left(Q_{4}\right)\right|=\left|\mathcal{P}_{3 n-3}\right|$ for all $n$, the map $\theta$ must be surjective as well.

\begin{tabular}{|c|c|c|}
\hline$n$ & $A v_{n}\left(Q_{4}\right)$ & $\mathcal{P}_{3 n-3}$ \\
\hline \multirow{7}{*}{2} & 12 & 123 \\
& 21 & 132 \\
& 123 & 124356 \\
& 213 & 124365 \\
3 & 132 & 123456 \\
& 231 & 123465 \\
& 312 & 132456 \\
& 321 & 132465 \\
\hline
\end{tabular}

Table 3: Sample values of $A v_{n}\left(Q_{4}\right)$ and their images under $\theta$ in $\mathcal{P}_{3 n}$

We note that our bijection is easily generalized:

Definition 49. Let $Q_{k, j}$ be the POP of size $k$ where $i<j$ for all $i, j \in[k]$ where $i \neq j$, as illustrated in Figure 6. 


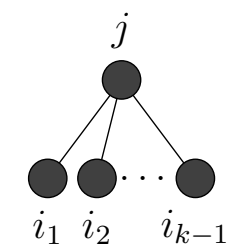

Figure 6: The POP $Q_{k, j}$, where $\left\{j, i_{1}, i_{2}, \ldots, i_{k-1}\right\}=[k]$

Theorem 50. There is a natural bijection between $A v_{n}\left(Q_{4, j}\right)$ and $\mathcal{P}_{3 n-3}$ for all $1 \leqslant j \leqslant 4$.

Proof. Due to the symmetry of $Q_{k, j}$ and the fact that $Q_{k}=Q_{k, 1}$, it is not hard to see that $\pi \in A v_{n}\left(Q_{k}\right)$ if and only if $\pi_{j} \pi_{[2, j-1]} \pi_{1} \pi_{[j+1, n]} \in A v_{n}\left(Q_{k, j}\right)$. We can then obtain a bijection from between $A v_{n}\left(Q_{4, j}\right)$ and $\mathcal{P}_{3 n-3}$ for all $1 \leqslant j \leqslant 4$ by composing $\theta$ from Theorem 48 with the bijection $\pi \mapsto \pi_{j} \pi_{[2, j-1]} \pi_{1} \pi_{[j+1, n]}$.

\section{Levels in compositions of $n$ of ones and twos}

Definition 51. Define $P_{k}$ to be a POP with $k$ elements where $1>3$. Its Hasse diagram is illustrated in Figure 7.

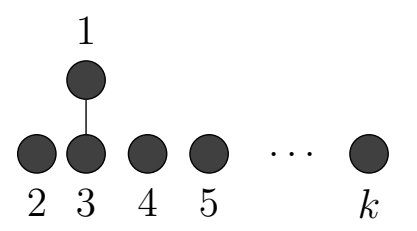

Figure 7: The POP $P_{k}$

Definition 52. A composition of $n$ is a way of writing $n$ as the sum of positive integers that sum to $n$, that is, an expression $i_{1}+i_{2}+\cdots+i_{k}=n$ for some $k \geqslant 1$. A composition of $n$ of ones and twos has the added restriction that $i_{j} \in\{1,2\}$ for all $j \in[n]$. In this paper, we will refer to a composition of $n$ of ones and twos simply as a "composition of $n "$ and denote the set of such compositions by $\mathcal{C}_{n}$.

Definition 53. A level in a composition of $n$ (of ones and twos) is a pair of consecutive ones or twos separated by a + sign. We define a marked composition of $n$ to be a composition of $n$ with exactly one level marked with a line above the pair of ones or twos. We may denote a single summand that is part of a level by including a line above it, i.e. $\overline{1}+\overline{1}=\overline{1+1}$ and $\overline{2}+\overline{2}=\overline{2+2}$. We denote the set of marked compositions of $n$ by $\mathcal{L}_{n}$.

We refer the reader to Table 4 for examples.

Gao and Kitaev [5] discovered that the sequence $\left|A v_{n}\left(P_{4}\right)\right|_{n \geqslant 1}$ corresponds to the OEIS sequence A045925 that enumerates the number of levels in all compositions of $n+1$ of ones and twos. In this section, we will demonstrate an explicit bijection between the two sets. To do so, we first construct an explicit bijection from $A v_{n}\left(P_{3}\right)$ to $\mathcal{C}_{n}$. 


\begin{tabular}{|c|c|c|c|c|}
\hline$n$ & $\left|\mathcal{C}_{n}\right|$ & $\mathcal{C}_{n}$ & $\left|\mathcal{L}_{n}\right|$ & $\mathcal{L}_{n}$ \\
\hline 1 & 1 & 1 & 0 & \\
\hline 2 & 2 & $1+1,2$ & 1 & $\overline{1+1}$ \\
\hline 3 & 3 & $\begin{array}{c}1+1+1 \\
2+1,1+2\end{array}$ & 2 & $\begin{array}{l}\overline{1+1}+1 \\
1+\overline{1+1}\end{array}$ \\
\hline 4 & 5 & $\begin{array}{c}1+1+1+1 \\
1+1+2, \\
1+2+1 \\
2+1+1 \\
2+2\end{array}$ & 6 & $\begin{array}{c}\overline{1+1}+1+1, \\
1+\overline{1+1}+1, \\
1+1+\overline{1+1}, \\
\overline{1+1+2} \\
2+\overline{1+1} \\
\frac{2+2}{2+2}\end{array}$ \\
\hline
\end{tabular}

Table 4: Marked compositions and levels of $n$ for small $n$

\subsection{Compositions of $n$}

Lemma 54. Let $F(n)$ denote the nth Fibonacci number, where $F(0)=0$ and $F(1)=1$. The size of $\mathcal{C}_{n}$ is $F(n)$ for all $n \geqslant 1$.

Proof. The size of $\mathcal{C}_{n}$ for $n=1$ and 2 can be verified easily. For $n \geqslant 3$, observe that

$$
c \in \mathcal{C}_{n-1} \Longleftrightarrow c+1 \in \mathcal{C}_{n} \text { and } c \in \mathcal{C}_{n-2} \Longleftrightarrow c+2 \in \mathcal{C}_{n}
$$

so $\left|\mathcal{C}_{n}\right|=\left|\mathcal{C}_{n-1}\right|+\left|\mathcal{C}_{n-2}\right|$, and $\left|\mathcal{C}_{n}\right|=F_{n}$.

Lemma 55. The size of $A v_{n}\left(P_{3}\right)$ is $F(n)$ for all $n \geqslant 1$. Moreover, all $n$-permutations avoiding $P_{3}$ for $n \geqslant 2$ are sum decomposable.

Proof. It is easy to see that $\left|A v_{1}\left(P_{3}\right)\right|=1=F(1)$ and $\left|A v_{2}\left(P_{3}\right)\right|=2=F(2)$. We refer the reader to Table 6 for examples. It is not difficult to see that for $n \geqslant 3$,

$$
\begin{gathered}
\sigma \in A v_{n-1}\left(P_{3}\right) \Longleftrightarrow 12[\sigma, 1] \in A v_{n}\left(P_{3}\right) \quad \text { and } \\
\sigma \in A v_{n-2}\left(P_{3}\right) \Longleftrightarrow 12[\sigma, 21] \in A v_{n}\left(P_{3}\right) .
\end{gathered}
$$

So by Theorem 12 ,

$$
\left|A v_{n}\left(P_{3}\right)\right|=\left|A v_{n-1}\left(P_{3}\right)\right|+\left|A v_{n-2}\left(P_{3}\right)\right|=F(n)+F(n-1)=F(n+1)
$$

for all $n \geqslant 3$ as well, proving the statements.

The previous two lemmas suggest that there is a natural bijection from the set $\mathcal{C}_{n}$ to $A v_{n}\left(P_{3}\right)$. Indeed there is, as we will see in the following theorem:

Theorem 56. Let $f: \mathcal{C}_{n} \rightarrow A v_{n}\left(P_{3}\right)$ defined as

$$
f\left(r_{1}+r_{2}+\cdots+r_{k}\right)=123 \cdots k\left[\alpha_{1}, \alpha_{2}, \ldots, \alpha_{k}\right]
$$

where $r_{1}+r_{2}+\cdots+r_{k}$ is a composition of $n$ of ones and twos, and

$$
\alpha_{i}= \begin{cases}1 & \text { if } r_{i}=1 \\ 21 & \text { if } r_{i}=2\end{cases}
$$

Then $f$ is a bijection. 


\begin{tabular}{|c|c|c|c|}
\hline$n$ & $F(n)$ & Compositions of $\boldsymbol{n}$ & Permutations in $\boldsymbol{A v}_{\boldsymbol{n}}\left(\boldsymbol{P}_{\mathbf{3}}\right)$ \\
\hline 1 & 1 & 1 & 1 \\
2 & 2 & $1+1$ & $12=12[1,1]$ \\
& & 2 & $21=21[1,1]$ \\
& & $1+1+1$ & $123=123[1,1,1]$ \\
3 & \multirow{3}{*}{3} & $1+2$ & $132=12[1,21]$ \\
& & $2+1$ & $213=12[21,1]$ \\
& & $1+1+1+1$ & $1234=1234[1,1,1,1]$ \\
4 & 5 & $2+1+1$ & $2134=123[21,1,1]$ \\
& & $1+1+2$ & $1243=123[1,1,21]$ \\
& & $1+2+1$ & $1324=123[1,21,1]$ \\
& & $2+2$ & $2143=12[21,21]$ \\
\hline
\end{tabular}

Table 5: Compositions of $n$ and their images under $f$ for small $n$

Proof. We refer the reader to Table 5 for examples for small $n$. First we check that if $r_{1}+r_{2}+\cdots+r_{k}$ is a composition of $n$, then $f\left(r_{1}+r_{2}+\cdots+r_{k}\right)$ is a permutation in $S_{n}$ :

$$
\begin{aligned}
\left|f\left(r_{1}+r_{2}+\cdots r_{k}\right)\right| & =\left|123 \cdots k\left[\alpha_{1}, \alpha_{2}, \ldots, \alpha_{k}\right]\right| \\
& =\left|\alpha_{1}\right|+\left|\alpha_{2}\right|+\cdots\left|\alpha_{k}\right| \\
& =r_{1}+r_{2}+\cdots r_{k} \\
& =n .
\end{aligned}
$$

Moreover, it is clear that if $\alpha_{i} \in\{1,21\}$ for all $i \in[k]$, then the permutation

$$
\pi=123 \cdots k\left[\alpha_{1}, \alpha_{2}, \ldots, \alpha_{k}\right]
$$

avoids $P_{3}$. It is easy to see that $f$ is injective by definition. By Lemmas 54 and $55, \mathcal{C}_{n}$ and $A v_{n}\left(P_{3}\right)$ both have $F(n)$ elements, so $f$ maps bijectively onto $A v_{n}\left(P_{3}\right)$.

\subsection{Levels of compositions of $n$}

\begin{tabular}{|c|c|c|c|c|}
\hline$n$ & $\left|A v_{n}\left(P_{3}\right)\right|$ & $A v_{n}\left(P_{3}\right)$ & $\left|A v_{n}\left(P_{4}\right)\right|$ & $A v_{n}\left(P_{4}\right)$ \\
\hline 1 & 1 & 1 & 1 & 1 \\
2 & 2 & 12,21 & 2 & 12,21 \\
& & $123,132,213$ & 6 & $123,132,213$, \\
3 & 3 & & & $231,312,321$ \\
& & & & $1234,1243,1324,1342$, \\
& 5 & 2134,2143 & 12 & $1423,1432,2134,2143$, \\
& & & & $2341,2431,3142,3241$ \\
\hline
\end{tabular}

Table 6: $A v_{n}\left(P_{3}\right)$ and $A v_{n}\left(P_{4}\right)$ for small $n$ 
Lemma 57. For $n \geqslant 1$, the size of the set $\mathcal{L}_{n}$ is $(n-1) F(n-1)$. Moreover, for $n \geqslant 3$, we can partition $\mathcal{L}_{n}$ into four sets:

$$
\begin{aligned}
& \left.A_{n}^{\prime}=\{\text { marked compositions of } n \text { that end with } 1 \text { (not } \overline{1})\right\} \\
& \left.B_{n}^{\prime}=\{\text { marked compositions of } n \text { that end with } 2 \text { (not } \overline{2})\right\} \\
& C_{n}^{\prime}=\{\text { marked compositions of } n \text { that end with } \overline{1+1}\} \\
& D_{n}^{\prime}=\{\text { marked compositions of } n \text { that end with } \overline{2+2}\}
\end{aligned}
$$

Proof. For $n \in[3]$, it is easy to check that $\left|L_{n}\right|=(n)-1 !=(n-1) F(n-1)$, as we show in Table 4 . For $n \geqslant 4$, we proceed by induction. Suppose that for some $k \geqslant 4$, the statement is true for all $n \in[k-1]$. It is clear that the union of $A_{k}^{\prime}, B_{k}^{\prime}, C_{k}^{\prime}$, and $D_{k}^{\prime}$ together make up $\mathcal{L}_{k}$, and that the following statements are true for all $n$ :

$$
\begin{aligned}
m \in \mathcal{L}_{n} & \Longleftrightarrow m+1 \in \mathcal{L}_{n+1}, & c \in \mathcal{C}_{n-1} & \Longleftrightarrow c+\overline{1+1} \in \mathcal{L}_{n+1} \\
m \in \mathcal{L}_{n-1} & \Longleftrightarrow m+2 \in \mathcal{L}_{n+1}, & c \in \mathcal{C}_{n-3} & \Longleftrightarrow c+\overline{2+2} \in \mathcal{L}_{n+1} .
\end{aligned}
$$

So by the inductive hypothesis,

$$
\left|A_{k}^{\prime}\right|=(k-2) F(k-2) \quad \text { and } \quad\left|B_{k}^{\prime}\right|=(k-3) F(k-3),
$$

and by Lemma 54,

$$
\left|C_{k}^{\prime}\right|=F(k) \quad \text { and } \quad\left|D_{k}^{\prime}\right|=F(k-2)
$$

Therefore the size of $\mathcal{L}_{k}$ is

$$
\begin{aligned}
& \left|A_{k}^{\prime}\right|+\left|B_{k}^{\prime}\right|+\left|C_{k}^{\prime}\right|+\left|D_{k}^{\prime}\right| \\
= & (k-1) F(k-1)+(k-2) F(k-2)+F(k)+F(k-2) \\
= & k F(k-1)-F(k-1)+k F(k-2)-2 F(k-2)+F(k)+F(k-2) \\
= & k(F(k-1)+F(k-2))-F(k-1)-F(k-2)+F(k) \\
= & k F(k),
\end{aligned}
$$

and the statement is true by induction on $n$.

Lemma 58. For $n \geqslant 1$, the size of $A v_{n}\left(P_{4}\right)$ is $n F(n)$. Moreover, for $n \geqslant 4$, we can partition $A v_{n}\left(P_{4}\right)$ into four sets. Specifically, $A v_{n}\left(P_{4}\right)=A_{n} \sqcup B_{n} \sqcup C_{n} \sqcup D_{n}$ where

$$
\begin{aligned}
& A_{n}:=\left\{12[1, \sigma]: \sigma \in A v_{n-1}\left(P_{4}\right)\right\}, \\
& B_{n}:=\left\{12[21, \sigma]: \sigma \in A v_{n-2}\left(P_{4}\right)\right\}, \\
& C_{n}:=\left\{21[\sigma, 1]: \sigma \in A v_{n-1}\left(P_{3}\right)\right\}, \quad \text { and } \\
& D_{n}:=\left\{3142[1,1, \sigma, 1]: \sigma \in A v_{n-3}\left(P_{3}\right)\right\} .
\end{aligned}
$$

Proof. For $n \leqslant 3$, it is easy to check that $\left|A v_{n}\left(P_{4}\right)\right|=n !=n F(n)$. We refer the reader to Table 6 for examples of

For $n \geqslant 4$, we may proceed by induction. First, we leave it to the reader to check that the following four claims are true for all $n \geqslant 4$ :

1. $12[1, \sigma] \in A v_{n}\left(P_{4}\right) \Longleftrightarrow \sigma \in A v_{n-1}\left(P_{3}\right)$ 
2. $12[21, \sigma] \in A v_{n}\left(P_{4}\right) \Longleftrightarrow \sigma \in A v_{n-2}\left(P_{3}\right)$,

3. $21[\sigma, 1] \in A v_{n}\left(P_{4}\right) \Longleftrightarrow \sigma \in A v_{n-1}\left(P_{3}\right), \quad$ and

4. $3142[1,1, \sigma, 1] \in A v_{n}\left(P_{4}\right) \Longleftrightarrow \sigma \in A v_{n-3}\left(P_{4}\right)$.

These imply that $A_{n}, B_{n}, C_{n}$ and $D_{n}$ are subsets of $A v_{n}\left(P_{4}\right)$.

Now suppose that for some $k \geqslant 4$, the statement is true for all $n \in[k-1]$. Then by our inductive hypothesis, we have that $A_{k}$ contains $(k-1) F(k-1)$ elements and $B_{k}$ contains $(k-2) F(k-2)$ elements.

By Lemma 55, $A v_{n}\left(P_{3}\right)$ is counted by the $(n+1)$ th Fibonacci number for all $n$, so $C_{k}$ contains $F(k)$ elements and $D_{k}$ contains $F(k-2)$ elements.

It is not hard to see that the four sets are disjoint, so the total number of items in the four sets is

$$
\begin{aligned}
& \left|A_{k}\right|+\left|B_{k}\right|+\left|C_{k}\right|+\left|D_{k}\right| \\
= & (k-1) F(k-1)+(k-2) F(k-2)+F(k)+F(k-2) \\
= & k F(k-1)-F(k-1)+k F(k-2)-2 F(k-2)+F(k)+F(k-2) \\
= & k(F(k-1)+F(k-2))-F(k-1)-F(k-2)+F(k) \\
= & k F(k) .
\end{aligned}
$$

It remains to check that any $k$-permutation avoiding $P_{4}$ indeed lies in $A_{k}, B_{k}, C_{k}$ or $D_{k}$. By Theorem 12, it suffices to show that

(a) 12, 21 and 3142 are the only simple permutations that avoid $P_{4}$,

(b) if $21[\alpha, \beta]$ avoids $P_{4}$ and $\alpha \neq 1$ is skew-sum indecomposable, then $\beta=1$, and

(c) if $12[\alpha, \beta]$ avoids $P_{4}$ and $\alpha$ is sum indecomposable, then $\alpha=1$ or 21 .

For (a), it is clear that 12 and 21 avoid $P_{4}$. Since 2413 contains $P_{4}$, any simple permutation of length at least 4 avoiding $P_{4}$ must contain 3142 by Theorem 11 . Let $\pi$ be such a simple permutation. We can view it as a lattice matrix. This is shown in Figure 8, with some alterations explained in the caption. The blocks $\alpha_{13}, \alpha_{14}, \alpha_{23}$ and $\alpha_{24}$ are adjacent to the bullet point representing the number 4 , so they must be trivial since $\pi$ is simple. Finally, $\alpha_{51}$ must be trivial, otherwise $\pi$ would be sum decomposable.

For (b), it is clear that $\beta$ can have size at most 1 , since otherwise $21[\alpha, \beta]$ would contain $P_{4}$ for any $|\alpha| \geqslant 2$.

For (c), suppose $\alpha$ is sum indecomposable and of length at least 3. Since $|\beta| \geqslant 1, \alpha$ must avoid $P_{3}$. By (a), $\alpha$ is an inflation of 21 or 3142. The only inflation of 21 avoiding $P_{3}$ is itself, while 3142 contains $P_{3}$. So $\alpha=1$ or 21 . 


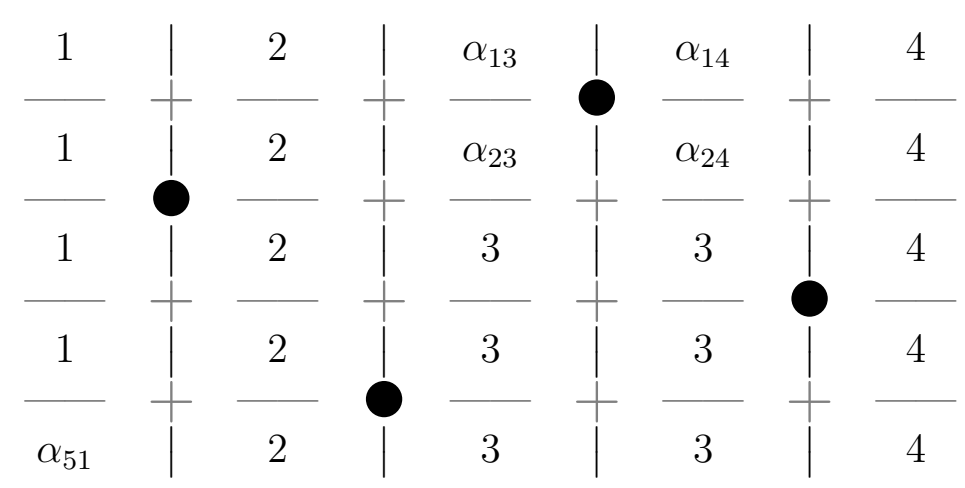

Figure 8: The lattice matrix $L_{3142}(\pi)$, with alterations. The 1 s corresponding to the pattern 3142 are replaced by bullet points. For all $i, j \in[5], \alpha_{i j}$ is replaced by some $\ell \in[4]$ if and only if $\pi$ would contain $P_{4}$ if $\alpha_{i j}$ were non-trivial and any point $\alpha_{i j}$ could take the place of the point labelled $\ell$ in the POP.

Once again, the previous two lemmas suggest that there is a natural bijection from the set $\mathcal{L}_{n+1}$ to $A v_{n}\left(P_{4}\right)$ - and indeed there is, as we will see in the main theorem of this section:

Theorem 59. Let $g: \mathcal{L}_{n+1} \rightarrow A v_{n}\left(P_{4}\right)$, where $n \geqslant 0$ and $r_{i} \in\{1,2, \overline{1}, \overline{2}\}$ for $i \in[k]$ and $k \geqslant 1$, defined as follows:

$$
g\left(r_{1}+r_{2}+\cdots r_{k}\right)= \begin{cases}1 & \text { if } k=1=r_{1}, \\ 21 & \text { if } k=2, \quad r_{1}=r_{2}=\overline{1}, \\ 12\left[1, g\left(r_{1}+\cdots+r_{k-1}\right)\right] & \text { if } r_{k}=1, \\ 12\left[21, g\left(r_{1}+\cdots+r_{k-1}\right)\right] & \text { if } r_{k}=2, \\ 21\left[f\left(r_{1}+\cdots+r_{k-2}\right), 1\right] & \text { if } r_{k-1}=r_{k}=\overline{1}, \\ 3142\left[1,1, f\left(r_{1}+\cdots+r_{k-2}\right), 1\right] & \text { if } r_{k-1}=r_{k}=\overline{2} .\end{cases}
$$

Then $g$ is a bijection.

Proof. We refer the reader to Tables 7 and 8 for enumerations of $\mathcal{L}_{n}$ and $A v_{n}\left(P_{4}\right)$ for small $n$, and Table 9 for the elements of $\mathcal{L}_{n+1}$ and their images under $g$ for small $n$. Recall from Theorem 56 that $f$ is a bijection, so it is clear from the definition of $g$ that it is injective. By Lemmas 57 and 58 , the sets $\mathcal{L}_{n+1}$ and $A v_{n}\left(P_{4}\right)$ both contain $n F(n)$ elements, so $g$ must be bijective. In addition, it can easily be seen that the inverse of $g$ is the following:

$$
g^{-1}(\pi)=\left\{\begin{array}{lll}
g^{-1}\left(\alpha_{2}\right)+\left|\alpha_{1}\right| & \text { if } & \pi=12\left[\alpha_{1}, \alpha_{2}\right] \\
f^{-1}(\alpha)+\overline{1+1} & \text { if } & \pi=21[\alpha, 1] \\
f^{-1}(\alpha)+\overline{2+2} & \text { if } & \pi=3142[1,1, \alpha, 1]
\end{array}\right.
$$

\section{$5 \quad$ Partial sums of signed displacements}

Definition 60. Let $R_{k}$ be the POP of size $k$ where $k>1$, as illustrated in Figure 9. 


\begin{tabular}{|c|c|c|c|}
\hline$n$ & $\left|\mathcal{L}_{n+1}\right|$ & $\begin{array}{l}\text { Number of compositions } \\
\text { in } \mathcal{L}_{n+1} \text { where the } \\
\text { last summand is not marked }\end{array}$ & $\begin{array}{l}\text { Number of compositions } \\
\text { in } \mathcal{L}_{n+1} \text { where the } \\
\text { last two terms are marked }\end{array}$ \\
\hline 1 & 1 & 0 & 1 \\
\hline 2 & 2 & 1 & 1 \\
\hline 3 & 6 & 3 & 3 \\
\hline 4 & 12 & 8 & 4 \\
\hline 5 & 25 & 18 & 7 \\
\hline 6 & 48 & 37 & 11 \\
\hline 7 & 91 & 73 & 18 \\
\hline 8 & 168 & 139 & 29 \\
\hline 9 & 306 & 259 & 47 \\
\hline 10 & 550 & 474 & 76 \\
\hline 11 & 979 & 856 & 123 \\
\hline$k$ & $k F(k)$ & $(k-1) F(k-1)+(k-2) F(k-2)$ & $L(k-1)=F(k-2)+F(k)$ \\
\hline
\end{tabular}

Table 7: Enumerating marked compositions for small $n$

\begin{tabular}{|c|c|c|c|}
\hline$n$ & $\left|A v_{n}\left(P_{4}\right)\right|$ & $\begin{array}{c}\text { Number of } \\
\text { sum decomposable } \\
n \text {-permutations avoiding } P_{4}\end{array}$ & $\begin{array}{c}\text { Number of } \\
\text { sum indecomposable } \\
n \text {-permutations avoiding } P_{4}\end{array}$ \\
\hline 1 & 1 & 0 & 1 \\
\hline 2 & 2 & 1 & 1 \\
\hline 3 & 6 & 3 & 3 \\
\hline 4 & 12 & 8 & 4 \\
\hline 5 & 25 & 18 & 7 \\
\hline 6 & 48 & 37 & 11 \\
\hline 7 & 91 & 73 & 18 \\
\hline 8 & 168 & 139 & 29 \\
\hline 9 & 306 & 259 & 47 \\
\hline 10 & 550 & 474 & 76 \\
\hline 11 & 979 & 856 & 123 \\
\hline$k$ & $k F(k)$ & $(k-1) F(k-1)+(k-2) F(k-2)$ & $L(k-1)=F(k-2)+F(k)$ \\
\hline
\end{tabular}

Table 8: Enumerating sum decomposable and sum indecomposable permutations in $A v_{n}\left(P_{4}\right)$ for small $n$

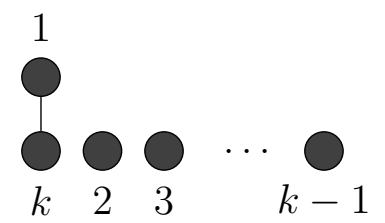

Figure 9: The POP $R_{k}$ 


\begin{tabular}{|c|c|c|c|c|c|}
\hline$n$ & $n F(n)$ & $\begin{array}{c}\text { Marked } \\
\text { compositions } \\
\text { of } n+1\end{array}$ & $\begin{array}{c}\text { Sum } \\
\text { decomposables } \\
\text { in } A v_{n}\left(P_{4}\right)\end{array}$ & $\begin{array}{c}\text { Marked } \\
\text { compositions } \\
\text { of } n+1\end{array}$ & $\begin{array}{c}\text { Sum } \\
\text { indecomposables } \\
\text { in } A v_{n}\left(P_{4}\right)\end{array}$ \\
\hline 1 & 1 & & & $\overline{1+1}$ & 1 \\
\hline 2 & 2 & $\overline{1+1}+1$ & $12=12[1,1]$ & $1+\overline{1+1}$ & $21=21[1,1]$ \\
\hline 3 & 6 & $\begin{array}{c}\overline{1+1}+1+1 \\
1+\overline{1+1}+1 \\
\overline{1+1}+2\end{array}$ & $\begin{array}{l}123=12[1,12] \\
132=12[1,21] \\
213=12[21,1]\end{array}$ & $\begin{array}{c}1+1+\overline{1+1} \\
2+\overline{1+1} \\
\overline{2+2}\end{array}$ & $\begin{array}{l}231=21[12,1] \\
321=21[1,21] \\
312=21[1,12]\end{array}$ \\
\hline 4 & 12 & $\begin{array}{c}\overline{1+1}+1+1+1 \\
1+\overline{1+1}+1+1 \\
1+1+\overline{1+1}+1 \\
\overline{1+1}+2+1 \\
2+\overline{1+1}+1 \\
\quad \overline{2+2}+1 \\
\overline{1+1}+1+2 \\
1+\overline{1+1}+2\end{array}$ & $\begin{array}{l}1234=12[1,123] \\
1243=12[1,132] \\
1324=12[1,213] \\
1342=12[1,231] \\
1432=12[1,321] \\
1423=12[1,312] \\
2134=12[21,12] \\
2143=12[21,21]\end{array}$ & $\begin{array}{c}1+1+1+\overline{1+1} \\
2+1+\overline{1+1} \\
1+2+\overline{1+1} \\
1+\overline{2+2}\end{array}$ & $\begin{array}{c}2341=21[123,1] \\
2431=21[132,1] \\
3241=21[213,1] \\
3142=3142[1,1,1,1]\end{array}$ \\
\hline
\end{tabular}

Table 9: Marked compositions of $n+1$ and their images under $g$ for small $n$

It is easy to see that the avoidance set of $R_{2}$ contains only the identity permutations. Observe that $R_{3}$ is equivalent to the POP $P_{3}$ introduced in Section 4 , where we showed that its avoidance set is enumerated by the well-known Fibonacci numbers. Gao and Kitaev [5] enumerated the avoidance sets of $R_{4}$ and $R_{5}$ in Theorems 14 and 33 of their paper, respectively. Moreover, they showed that for all $k \geqslant 3$, the avoidance set of $R_{k}$ is in one-to-one correspondence with $n$-permutations such that for each cycle $c$, the smallest integer interval containing all elements of $c$ has at most $k-1$ elements.

They also observed that $A v_{n}\left(R_{4}\right)$ and the set of $n$-permutations for which the partial sums of signed displacements do not exceed 2 (to be defined later) are of the same size for all $n \geqslant 1$, and asked if there is an interesting bijection between the sets. We construct such a bijection in this section by analyzing the two sets in detail.

\subsection{Analysing $A v_{n}\left(R_{4}\right)$}

Theorem 61. If $\pi$ is an n-permutation that avoids $R_{4}$ and $n \geqslant 5$, then $\pi$ is sum decomposable. Moreover, if $12[\alpha, \beta]$ avoids $R_{4}$ for permutations $\alpha$ and $\beta$ where $\alpha$ is sum indecomposable, then $\alpha$ is 1, 21, 231, 321, 312 or 2413.

Proof. We claim that the only simple permutations avoiding $R_{4}$ are 1, 12, 21 and 2413 . Recall that any simple $n$-permutation contains the patterns 132, 213 and 312 if $n \geqslant 4$. Thus, we can write such a simple permutation as the string of factors

$$
\alpha^{(1)} p \alpha^{(2)} q \alpha^{(3)} r \alpha^{(4)}
$$

where $\alpha_{i}$ are possibly empty factors (of length 0 ) and $p, q$ and $r$ are factors of length 1 where $\operatorname{red}(p q r)=312$. It is clear that if $\alpha^{(2)}$ and $\alpha^{(3)}$ were not empty, if $\alpha^{(1)}>p$ or if 
$\alpha^{(4)}<p$ then the permutation would contain $R_{4}$. So $\alpha^{(2)}$ and $\alpha^{(3)}$ are empty and if $\alpha^{(1)}$ and $\alpha^{(4)}$ are not empty then we must have $\alpha^{(1)}<p<\alpha^{(4)}$. However, this implies that if $\alpha_{4}$ is not empty then the permutation must be sum decomposable, so $\alpha^{(4)}$ is empty. If $\left|\alpha^{(1)}\right|=t \geqslant 2$, then we must have $\alpha_{[1, t-1]}^{(2)}<q$ otherwise the permutation contains $R_{4}$. This forces $\alpha_{[1, t-1]}^{(1)}$ to be the interval [t-1] which would mean that the permutation is sum decomposable. So $\left|\alpha^{(1)}\right|=1$ if it is not empty. In this case we must have $q<\alpha^{(1)}<r$, which yields the permutation 2413 .

Finally we show that all $n$-permutations avoiding $R_{4}$ are sum decomposable for $n \geqslant 5$. If $21\left[\beta_{1}, \beta_{2}\right]$ avoids $R_{4}$, then $\beta_{1}$ and $\beta_{2}$ must have lengths 1 or 2 . If $2413\left[\beta_{1}, \beta_{2}, \beta_{3}, \beta_{4}\right]$ avoids $R_{4}$, then each $\beta_{i}$ must be of length exactly 1 for all $i \in$ [4]. The only sum indecomposable permutations avoiding $R_{4}$ are $1,21,321,312,231$ and 2413 , so $\alpha$ in the theorem must be one of these.

Corollary 62. Let $a_{n}:=\left|A v_{n}\left(R_{4}\right)\right|$. Then

$$
a_{n}= \begin{cases}n ! & \text { if } 1 \leqslant n \leqslant 3 \\ 12 & \text { if } n=4, \\ a_{n-1}+a_{n-2}+3 a_{n-3}+a_{n-4} & \text { if } n \geqslant 5 .\end{cases}
$$

Proof. The formula is clear for $n \leqslant 3$. Since $R_{4}$ represents a set of 12 permutations, $A v_{4}\left(R_{k}\right)=4$ ! $-12=12$. For $n \geqslant 5$, recall that if $\pi=12[\alpha, \beta]$ for some permutations $\alpha$ and $\beta$ where $\alpha$ is sum indecomposable, then $\alpha$ and $\beta$ are unique by Theorem 12. The recursive formula then follows directly from Theorem 61 .

\subsection{Partial sums of signed displacements}

Definition 63. Let $\pi$ be an $n$-permutation and $i, j \in[n]$. The $j$ th signed displacement of $\pi$ is defined as $\pi_{j}-j$. The $i$ th partial sum of signed displacements of $\pi$ (denoted $\pi_{\Sigma}^{i}$ ) is the sum of the first $i$ signed displacements of $\pi$. That is, $\pi_{\Sigma}^{i}:=\sum_{j=1}^{i} \pi_{j}-j$.

We denote the set of $n$-permutations for which the partial sums of signed displacements do not exceed 2 by $\mathfrak{S}_{n}$. For examples, refer to Figures 10 and 11.

$$
\begin{array}{c|cccc}
\pi_{j} & 3 & 1 & 4 & 2 \\
j & 1 & 2 & 3 & 4 \\
\pi_{j}-j & 2 & -1 & 1 & -2 \\
\hline \text { partial sum } & 2 & 1 & 2 & 0
\end{array}
$$

Figure 10: A permutation in $\mathfrak{S}_{4}$

$$
\begin{array}{c|cccc}
\pi_{j} & 4 & 1 & 3 & 2 \\
j & 1 & 2 & 3 & 4 \\
\pi_{j}-j & 3 & -1 & 0 & -2 \\
\hline \text { partial sum } & 3 & 2 & 2 & 0
\end{array}
$$

Figure 11: A permutation that is not in $\mathfrak{S}_{4}$ 
Lemma 64. For an n-permutation $\pi$ and $k \in[n]$, we have $\pi_{\Sigma}^{k}=0$ if and only if $\pi_{[1, k]}$ is itself a k-permutation (without being reduced).

Proof. The sum of numbers in $[k]$ under any permutation is invariant, so $\sigma_{\Sigma}^{k}=0$ for any $k$-permutation $\sigma$. Now suppose that $\pi_{\Sigma}^{k}=0$ for an $n$-permutation $\pi$ and $k \in[n]$. That is, $\sum_{j=1}^{k}\left(\pi_{j}-j\right)=0$. Let $i_{1}, i_{2}, \ldots, i_{k}$ be integers in $[k]$ such that $1 \leqslant \pi_{i_{1}}<\pi_{i_{2}}<\cdots<$ $\pi_{i_{k}} \leqslant n$. Observe that $\pi_{i_{j}}-j$ is non-negative for all $j \in[k]$. The only way they can sum to 0 is if $\pi_{i_{j}}-j=0$ for all $j \in[k]$. Therefore $\left\{\pi_{i} \mid i \in[k]\right\}=[k]$ which means that $\pi_{[1, k]}$ is a $k$-permutation.

The proof of the following theorem was inspired by the proof on OEIS page on A214663.

Theorem 65. If $\pi \in \mathfrak{S}_{n}$ and $n \geqslant 5$, then $\pi$ must be of the form $12[\alpha, \beta]$, where

$$
\beta \in\{1,21,231,312,321,3142\}
$$

and $\alpha$ is a conforming permutation $\alpha$ of length $n-|\beta|$.

Proof. For $n \geqslant 5$, observe that the number $n$ can only occur as one of the last 3 terms of a conforming $n$-permutation. Note also that the sum of two conforming permutations is conforming. Let $\pi$ be a conforming $n$-permutation. Then we have the following cases:

- Case 1: $\pi_{n}=n$. So $\pi_{n}-n=0$, and $\pi$ is conforming if and only if $\pi_{\Sigma}^{k}$ does not exceed 2 for all $k \in[n-1]$. That is, $\pi$ is of the form $\alpha \oplus 1$ where $\alpha$ is a conforming $(n-1)$-permutation.

- Case 2: $\pi_{n-1}=n$. So $\pi_{n-1}-(n-1)=1$, and $\pi$ is conforming only if $\pi_{\Sigma}^{n-2}=1$ or 0 . We can further break down into cases:

(a) If $\pi_{\Sigma}^{n-2}=0$, then $\pi_{[1, n-2]}$ must itself be an $(n-2)$-permutation by Lemma 64 . Then we must have $\pi_{n}=n-1$ - that is, $\pi$ is of the form $\alpha \oplus 21$ where $\alpha$ is a conforming $(n-2)$-permutation.

(b) If $\pi_{\Sigma}^{n-2}=1$ then $\pi_{\Sigma}^{n-1}=2$, so $\pi_{n}=n-2$.

- If $\pi_{\Sigma}^{n-3}=0$, then $\pi_{[1, n-3]}$ must itself be an $(n-3)$-permutation by Lemma 64 . Since $\pi_{n-2}=n-1$ and $\pi_{n}=n-2$, so $\pi$ must be of the form $\alpha \oplus 231$ where $\alpha$ is a conforming $(n-3)$-permutation.

- If $\pi_{\Sigma}^{n-3}=1=\pi_{\Sigma}^{n-2}$, then $\pi_{n-2}=n-2$, which is impossible since $\pi_{n}=n-2$.

- If $\pi_{\Sigma}^{n-3}=2$. then $\pi_{n-2}=n-3$. Since the partial sums of signed displacements of $\pi$ cannot exceed 2 and $\pi_{[n-2, n]}=(n-3) n(n-2)$, we must have $\pi_{n-3}=n-1$. This means that $\pi_{\Sigma}^{n-4}=0$. Then $\pi_{[1, n-4]}$ must itself be an $(n-4)$-permutation by Lemma 64 , and $\pi$ is of the form $\alpha \oplus 3142$ where $\alpha$ is a conforming $(n-4)$-permutation.

- Case 3: $\pi_{n-2}=n$. So $\pi_{n-2}-(n-2)=2$, and $\pi$ is conforming if and only if the $(n-2)$ th partial sum of signed displacements is 0 . By Lemma 64 , the factor $\pi_{[1, n-3]}$ must be an $(n-3)$-permutation itself. It is easy to check that 321 and 312 are both conforming, so $\pi$ is of the form $\alpha \oplus 321$ or $\alpha \oplus 312$, where $\alpha$ is a conforming $(n-3)$-permutation. 
Corollary 66. Let $b_{n}:=\left|\mathfrak{S}_{n}\right|$. Then

$$
b_{n}= \begin{cases}n ! & \text { if } 1 \leqslant n \leqslant 3 \\ 12 & \text { if } n=4, \\ b_{n-1}+b_{n-2}+3 b_{n-3}+b_{n-4} & \text { if } n \geqslant 5\end{cases}
$$

Proof. It is easy to check that all $n$-permutations are conforming for $1 \leqslant n \leqslant 3$. For $n=4$, there are exactly twelve conforming $n$-permutations, namely $1234,1243,1324$, 1342, 1423, 1432, 2134, 2143, 2314, 3124, 3142 and 3214. For $n \geqslant 5$, the recursive formula for $b_{n}$ follows directly from Theorem 65 .

\subsection{The bijection}

Theorem 67. Define $\theta: A v_{n}\left(R_{4}\right) \rightarrow \mathfrak{S}_{n}$ as

$$
\theta(\pi)= \begin{cases}\pi & \text { if }|\pi| \in[4], \\ \beta \oplus 3142 & \text { if } \pi=2413 \oplus \beta \text { for some permutation } \beta, \text { and } \\ \beta \oplus \alpha & \text { if } \pi=\alpha \oplus \beta \text { for some permutations } \alpha \text { and } \beta \\ & \text { where } \alpha \neq 2413 .\end{cases}
$$

Then $\theta$ is a bijection.

Proof. We know that $\theta$ is indeed a function from $A v_{n}\left(R_{4}\right)$ to $\mathfrak{S}_{n}$ by Theorems 61 and 65 . It is clear from the definition that $\theta$ is an injection. Corollaries 62 and 66 demonstrate that $\left|\mathfrak{S}_{n}\right|=\left|A v_{n}\left(R_{4}\right)\right|$ for all $n \geqslant 1$, so $\theta$ must be a bijection.

\section{Simple permutations avoiding 2413, 3412 and 3421}

Albert and Atkinson [2] proved that a permutation class with only finitely many simple permutations has a readily computable algebraic generating function and has a finite basis. So far, we have been dealing mostly with avoidance sets that contain only finitely many simple permutations. In this chapter, we will show an example of a permutation class with a finite basis that contains infinitely many simple permutations as well as construct an algorithm that allows us to obtain the entire set recursively.

Definition 68. Let $P$ be a pattern, a set of patterns, or a POP. Denote $A v_{n}^{S}(P)$ as the set of simple $n$-permutations avoiding $P$.

Theorem 69. The set of simple permutations avoiding $P:=\{2413,3412,3421\}$ is enumerated by a translate of the well-known Fibonacci sequence. Specifically, if $n \geqslant 3$, then $\left|A v_{n}^{S}(P)\right|=F(n-3)$ where $F(0)=0, F(1)=1$ and $F(n)=F(n-1)+F(n-2)$ for all $n \geqslant 3$.

\subsection{Partitioning the simple permutations into 3 sets}

In order to enumerate the set of simple permutations avoid 2413, 3412 and 3421, we will identify the types of permutations that appear in it. 
Lemma 70. Let $\pi$ be a simple $n$-permutation that avoids $P$ for some $n \geqslant 4$. Then $\pi_{n-1}=n$ and $\pi_{1}=n-1$.

Proof. We first prove that $\pi_{n-1}=n$. Let $j$ and $k$ be in $[n]$ such that $\pi_{k}=n$ and $\pi_{j}:=\max \left(\left\{\pi_{1}, \pi_{2}, \ldots, \pi_{k-1]}\right\}\right)$. Suppose there exists some $\ell>k$ such that $\pi_{\ell}<\pi_{j}$. Note that $\operatorname{red}\left(\pi_{j} \pi_{k} \pi_{\ell_{1}} \pi_{\ell_{2}}\right)$ would be 3412 or 3421 if $\ell_{1}<\ell_{2}$ and both $\ell_{1}$ and $\ell_{2}$ both satisfied conditions for $\ell$. So $\ell$ must be unique. If $\ell \neq n$ then $\pi_{j}<\pi_{n}$ so $\pi_{j} \pi_{k} \pi_{\ell} \pi_{n}=\pi_{j} n \pi_{\ell} \pi_{n}$ reduces to 2413. So $\ell=n$. We know that if $i>k$ and $i \neq \ell$ then $\pi_{i}>\pi_{j}$, which implies that $\pi_{[k, n-1]}$ is an interval. Since $\pi$ is simple and $k>1$, we must have $k=n-1$.

Next, we prove that $\pi_{1}=n-1$. Let $\pi$ be represented as the string of factors $\alpha(n-$ 1) $\beta n k$ where $k \in[n-2]$. We know that $\beta$ cannot be empty, since then $(n-1) n$ would be an nontrivial interval in $\pi$. Suppose $\alpha$ is not empty. Note that the subsequence $\hat{\alpha} \hat{\beta} k$ must reduce to 123 or 132 or 231 for all $\hat{\alpha} \in \alpha$ and $\hat{\beta} \in \beta$ by elimination (since $n-1$ is larger than the three points and $\hat{\alpha}(n-1) \hat{\beta} k$ cannot reduce to 2413,3412 or 3421$)$. The set of patterns $\{123,132,231\}$ is equivalent to the POP of size three where $1<2$, so the above observation implies that $\alpha<\beta$. If $\alpha>k$ then $\pi$ would be skew sum decomposable, while if $\alpha<k$ then $\pi$ would be sum decomposable, both of which contradict the simpleness of $\pi$. But this implies that $\beta>k$ which makes $(n-1) \beta n$ a nontrivial interval of $\pi$. So $\alpha$ must be empty, meaning that $\pi_{1}=n-1$.

Lemma 71. Let $\pi$ be a simple $n$-permutation that avoids $P$ where $n \geqslant 5$. Then we have exactly three cases:

$$
\begin{aligned}
& \text { 1. } \pi_{2}=1 \text { and } \pi_{3}=n-2 \text {, } \\
& \text { 2. } \pi_{2}=n-3 \text { and } \pi_{n-2}=n-2 \text {, or } \\
& \text { 3. } \pi_{2}=1, \pi_{3}=n-3 \text { and } \pi_{n-2}=n-2 \text {. }
\end{aligned}
$$

Proof. We know from Lemma 70 that $\pi_{n-1}=n$ and $\pi_{1}=n-1$. Write $\pi$ as the string of factors $(n-1) \gamma n k$ where $k$ has length 1 . If $k=n-2$ then $\gamma$ is an interval of length $n-3 \geqslant 2$. So $k \in[n-3]$ and $n-2 \in \gamma$. Let $s:=|\gamma|$ and $t \in[s]$ such that $\gamma_{t}=n-2$.

Suppose $t<s$. We want to show that $t=2$ and $\gamma_{1}=1$, which will satisfy case a. If $t=1$ then $\pi_{[1,2]}=(n-1)(n-2)$ is a nontrivial interval, so $t>1$. So $t \in[2, s-1]$. Note that $\gamma_{i}(n-2) \gamma_{j} k$ reduces to 1423,1432 or 2431 for all $1 \leqslant i<t<j \leqslant s$ by elimination since $n-2$ is the largest of the four points, and the subsequence cannot reduce to 2413 , 3412 or 3421 . This observation implies that $\gamma_{[1, t-1]}<\gamma_{[t+1, s-1]}$. So $\gamma_{[1, t-1]}<\gamma_{[t, s]}$. If $k<\gamma_{[t, s]}$ then $\gamma_{[t, s]}$ is a nontrivial interval. So $\gamma_{[1, t-1]}<k$ which means that $\gamma_{[1, t-1]}$ is an interval. By the simpleness of $\pi$ we have $t=2$. Since $\gamma_{[1, t-1]}=\gamma_{1}$ is smaller than all the other points, $\gamma_{1}=1$.

Now suppose that $t=s$. That is, $\pi_{n-2}=n-2$. If $k=n-3$ then $\gamma$ is an interval of length 1 . This is only possible if $\pi=41352$, which satisfies case a. So if $n \geqslant 6$ then $n-3$ is in $\gamma$ instead of $k$. Let $n \geqslant 6$ and $m \in[s]$ such that $\gamma_{m}=n-3$. If $m=1$ then $\pi$ satisfies case b. So suppose $m>1$. We know that $m \neq s-1$ since that would mean that $\gamma_{[s-1, s]}=(n-3)(n-2)$ is a nontrivial interval in $\pi$. So $m \in[2, s-2]$. Note 
that $\gamma_{i}(n-3) \gamma_{j} k$ reduces to 1423,1432 or 2431 for all $1 \leqslant i<m<j \leqslant s-1$, since $n-3$ is the largest of the four points and the subsequence cannot reduce to 2413,3412 or 3421. This observation implies that $\gamma_{[1, m-1]}<\gamma_{[m+1, s-1]}$. So $\gamma_{[1, m-1]}<\gamma_{[m, s]}<n-1$. If $k<\gamma_{[m, s]}$ then $\gamma_{[m, s]}$ is a nontrivial interval. So $\gamma_{[1, m-1]}<k$ which means that $\gamma_{[1, m-1]}$ is an interval. By the simpleness of $\pi$ we have $m=2$. Since $\gamma_{[1, m-1]}=\gamma_{1}$ is smaller than all the other points, $\gamma_{1}=1$.

By Lemmas 70 and 71, we can partition the set of simple permutations of length $n$ avoiding $P$ by their initial terms.

Definition 72. For $n \geqslant 4$, define $A_{n}, B_{n}$ and $C_{n}$ to be the set of simple $n$-permutations avoiding $P$ beginning with $(n-1) 1(n-2),(n-1)(n-3)$ and $(n-1) 1(n-3)$, respectively. Denote their cardinalities by $a_{n}, b_{n}$ and $c_{n}$.

Remark 73. The above definition holds for all $n \geqslant 4$ even if $A_{n}, B_{n}$ or $C_{n}$ are empty. Table 6.1 summarizes the types of permutations in these sets due to Lemmas 70 and 71 . Table 12 gives sample values of $a_{n}, b_{n}$ and $c_{n}$ for $4 \leqslant n \leqslant 8$.

\begin{tabular}{|c|cc|}
\hline Set & Permutations in the set are of the form \\
\hline$A_{n}$ & $(n-1) 1(n-2) \cdots n k$ & where $k \in[2, n-3]$ \\
$B_{n}$ & 3142 or $(n-1)(n-3) \cdots(n-2) n k$ & where $k \in[2, n-4]$ \\
$C_{n}$ & $(n-1) 1(n-3) \cdots(n-2) n k$ & where $k \in[2, n-4]$ \\
\hline
\end{tabular}

Table 10: Summary of the types of permutations in $A v_{n}^{S}(2413,3412,3421)$ for $n \geqslant 4$. The ellipses represent (possibly empty) factors of the permutations.

\subsection{Recursive functions}

We will show that for all $n \geqslant 6$, we can obtain simple permutations of length $n$ that avoid $P$ by adding points to smaller simple permutations that avoid $P$.

Definition 74. For $n \geqslant 6$, let $S_{n}$ denote the set of permutations on [n], and let

$$
f_{A}: A v_{n-2}^{S}(P) \rightarrow S_{n}, \quad f_{B}: A v_{n-2}^{S}(P) \rightarrow S_{n}, \quad \text { and } \quad f_{C}: B_{n-1} \rightarrow S_{n},
$$

be functions defined as follows:

$$
\begin{aligned}
& f_{A}\left(\pi_{1} \pi_{2} \cdots \pi_{n-2}\right):=(n-1) 1\left(\pi_{1}+1\right)\left(\pi_{2}+1\right) \cdots\left(\pi_{n-3}+2\right)\left(\pi_{n-2}+1\right), \\
& f_{B}\left(\pi_{1} \pi_{2} \cdots \pi_{n-2}\right):=(n-1) \pi_{1} \pi_{2} \cdots \pi_{n-3} n \pi_{n-2}, \quad \text { and } \\
& f_{C}\left(\pi_{1} \pi_{2} \cdots \pi_{n-1}\right):=\left(\pi_{1}+1\right) 1\left(\pi_{2}+1\right) \cdots\left(\pi_{n-1}+1\right) .
\end{aligned}
$$

Definition 75. For $n \geqslant 6$, let

$$
g_{A}: A_{n} \rightarrow S_{n-2}, \quad g_{B}: B_{n} \rightarrow S_{n-2}, \quad \text { and } \quad g_{C}: C_{n} \rightarrow S_{n-1},
$$

be functions defined as

$$
\begin{aligned}
& g_{A}(\sigma):=\left(\sigma_{3}-1\right)\left(\sigma_{4}-1\right) \cdots\left(\sigma_{n-2}-1\right)\left(\sigma_{n-1}-2\right)\left(\sigma_{n}-1\right), \\
& g_{B}(\sigma):=\sigma_{2} \sigma_{3} \cdots \sigma_{n-2} \sigma_{n} \\
& g_{C}(\sigma):=\left(\sigma_{1}-1\right)\left(\sigma_{3}-1\right)\left(\sigma_{4}-1\right) \cdots\left(\sigma_{n}-1\right) .
\end{aligned}
$$


where $\sigma=\sigma_{1} \sigma_{2} \cdots \sigma_{n} \in A v_{n}^{S}(P)$. Note that $g_{A}(\sigma)=\operatorname{red}\left(\sigma_{[3, n]}\right), g_{B}(\sigma)=\operatorname{red}\left(\sigma_{[2, n-1]} \sigma_{n}\right)$ and $g_{C}(\sigma)=\operatorname{red}\left(\sigma_{1} \sigma_{[3, n]}\right)$.

Lemma 76. If $\pi \in A v_{n-2}^{S}(P)$ and $n \geqslant 6$, then $f_{A}(\pi)$ is simple and avoids $P$.

Proof. Recall that $f_{A}: A v_{n-2}^{S}(P) \rightarrow A v_{n}^{S}(P)$ and

$$
f_{A}\left(\pi_{1} \pi_{2} \cdots \pi_{n-2}\right):=(n-1) 1\left(\pi_{1}+1\right)\left(\pi_{2}+1\right) \cdots\left(\pi_{n-3}+2\right)\left(\pi_{n-2}+1\right) .
$$

Suppose that $f_{A}(\pi)_{[i, j]}$ is an interval for some $i, j \in[n]$. The following cases show that all intervals in $f_{A}(\pi)$ are of length 0,1 or $n$ thereby proving that $f_{A}(\pi)$ is simple:

- Case 1: $i=1$. Recall that $f_{A}(\pi)_{1}=n-1$ and $f_{A}(\pi)_{2}=1$, so if $j \geqslant 2$, then $f_{A}(\pi)_{[i, j]}$ must contain all the numbers in $[n-1]$ and so must have length at least $n-1$. Observe that $f_{A}(\pi)_{n-1}=\pi_{n-3}+2=n$. So $f_{A}(\pi)_{[1, j]}$ must contain $[n]$, i.e. $j=n$.

- Case 2: $i=2$. Observe that $f_{A}(\pi)_{2}=1$, so $f_{A}(\pi)_{[2, j]}$ is the interval $[m]$ for some $m \in[n]$. If $j>2$, then $f_{A}(\pi)_{[3, j]}$ is the interval $[2, m]$. Recall that $f_{A}(\pi)_{[3, j]}=$ $\left(\pi_{1}-1\right)\left(\pi_{2}-1\right) \cdots\left(\pi_{j-2}-1\right)$, so $\pi_{[1, j-2]}$ is an interval as well, and is a trivial interval only if $j \leqslant 3$. We know that $f_{A}(\pi)_{[2,3]}=1(n-3)$ is not an interval, so $j \leqslant 2$.

- Case 3: $i \geqslant 3$. If $j<n-1$ then $f_{A}(\pi)_{[i, j]}=\left(\pi_{i-2}-1\right)\left(\pi_{i-1}-1\right) \cdots\left(\pi_{j-2}-1\right)$. So $f_{A}(\pi)_{[i, j]}$ is an interval only if $\pi_{[i-2, j-2]}$ is an interval, which is true only if $j \leqslant i$ since $j-2<n-3$. Note that $f_{A}(\pi)_{n-1}=\pi_{n-3}+2=n$. So if $j \geqslant n-1$ then $f_{A}(\pi)_{[i, j]}$ contains the point $n$. This means that $f_{A}(\pi)_{[i, j]}$ can only be a nontrivial interval if it contains $\pi_{1}=n-1$ as well, which is impossible since $i \geqslant 3$. So $f_{A}(\pi)_{[i, j]}$ is an interval only if $j \leqslant i$.

Finally, we check that $f_{A}(\pi)$ avoids $P$. Suppose we have $1 \leqslant i<j<k<\ell \leqslant n$ such that $f_{A}(\pi)_{i} f_{A}(\pi)_{j} f_{A}(\pi)_{k} f_{A}(\pi)_{\ell}$ reduces to a pattern in $P$. Since $\pi$ avoids $P$, such a subsequence must contain the numbers $n-1$ or 1 . However, the only term larger than $n-1$ is $n=\pi_{n-3}+2=f_{A}(\pi)_{n-1}$ by Lemma 70 , while all patterns in $P$ have the largest term 4 as the third last number in the pattern, so it cannot contain the number $n-1$. On the other hand, 1 is the second term of $f_{A}(\pi)$, but is the third or fourth term in the patterns in $P$. So it cannot contain 1 either. So $f_{A}(\pi)$ avoids $P$.

Lemma 77. If $\pi \in A v_{n-2}^{S}(P)$ and $n \geqslant 6$, then $f_{B}(\pi)$ is simple and avoids $P$.

Proof. Recall that $f_{B}: A v_{n-2}^{S}(P) \rightarrow A v_{n}^{S}(P)$, and

$$
f_{B}\left(\pi_{1} \pi_{2} \cdots \pi_{n-2}\right):=(n-1) \pi_{1} \pi_{2} \cdots \pi_{n-3} n \pi_{n-2} .
$$

Suppose that $f_{B}(\pi)_{[i, j]}$ is an interval for some $i, j \in[n]$. The following cases show that all intervals in $f_{B}(\pi)$ are of length 0,1 or $n$ thereby proving that $f_{B}(\pi)$ is simple:

- Case 1: $2 \leqslant i, j \leqslant n-2$. Observe that $f_{B}(\pi)_{[i, j]}=\pi_{[i-1, j-1]}$ is an interval only if $i \geqslant j$ since $\pi$ is simple. So $i \geqslant j$. 
- Case 2: $i=1 \leqslant j \leqslant n-2$. Observe that $f_{B}(\pi)_{\ell}<f_{B}(\pi)_{1}=n-1$ for all $\ell \in[2, j]$. So the factor $f_{B}(\pi)_{[2, j]}=\pi_{[1, j-1]}$ must also be interval. Since $\pi$ is simple, and $j-1<n-2$, the factor $\pi_{[1, j-1]}$ is an interval only if $j \leqslant 2$. We know that $f_{B}(\pi)_{[1,2]}=(n-1) \pi_{1}=(n-1)(n-3)$ is not an interval, so we must have $j=1$.

- Case 3: $n-1 \leqslant j$. Observe that $f_{B}(\pi)_{n-1}=n$. If $i<j$ then $f_{B}(\pi)_{[i, j]}$ must also contain $n-1=f_{B}(\pi)_{1}$, so $i=1$. So it is a factor of length at least $n-1$. But $f_{B}(\pi)_{[1, n-1]}$ is not an interval since it omits $f_{B}(\pi)_{n}=\pi_{n-2}$ which is in $[2, n-2]$. So implies that $i=1$ and $j=n$. Otherwise $i \geqslant j$.

Finally, we prove that $f_{B}(\pi)$ avoids $P$. Suppose we have $1 \leqslant i<j<k<\ell \leqslant n$ such that $f_{B}(\pi)_{i} f_{B}(\pi)_{j} f_{B}(\pi)_{k} f_{B}(\pi)_{\ell}$ reduces to a pattern in $P$. Then such a subsequence must contain the number $n-1=f_{B}(\pi)_{1}$ or $n=f_{B}(\pi)_{n-1}$ since we know that $\pi$ avoids $P$. Clearly it cannot contain the number $n$ since it is the second last term in the permutation, but 4 never occurs as the last or second last term in patterns in 2413, 3412 or 3421 . Then we must have $i=1$. But no patterns in $P$ start with 4 , so $f_{B}(\pi)$ indeed avoids $P$.

Lemma 78. If $\pi \in B_{n-1}$ and $n \geqslant 6$, then $f_{C}(\pi)$ is simple and avoids $P$.

Proof. Recall that $f_{C}: B_{n-1} \rightarrow A v_{n}^{S}(P)$ and

$$
f_{C}\left(\pi_{1} \pi_{2} \cdots \pi_{n-1}\right):=\left(\pi_{1}+1\right) 1\left(\pi_{2}+1\right) \cdots\left(\pi_{n-1}+1\right) .
$$

It is easy to see that $f_{C}(\pi)$ avoids $P$. The only way that $f_{C}(\pi)$ could contain $P$ due to the addition of the point 1 is if there are at least 2 terms preceding it. However, it is inserted in the second position, so $f_{C}(\pi)$ does not contain $P$.

Suppose that $f_{C}(\pi)_{[i, j]}$ is an interval for some $i, j \in[n]$. The following cases show that all intervals in $f_{B}(\pi)$ are of length 0,1 or $n$ thereby proving that $f_{C}(\pi)$ is simple:

- Case 1: $i=1$. Suppose $j \geqslant 2$. Observe that $f_{C}(\pi)_{1}=\pi_{1}+1=n-1$ and $f_{C}(\pi)_{2}=1$. Then the interval $f_{C}(\pi)_{[i, j]}$ must contain all the numbers in $[n-1]$. This includes $f_{C}(\pi)_{n}=\pi_{n-1}+1$ which is in [3,n-4] for $n \geqslant 6$. This is because $\pi_{n-1}$ cannot be $n-4, n-3$ or $n-2$ by Lemmas 70 and 71 , and is not 1 or $n-1$ since $\pi$ is simple. So $j=n$.

- Case 2: $i=2$. Observe that $f_{C}(\pi)_{2}=1$ and $f_{C}(\pi)_{3}=\pi_{2}+1=n-3$. If $j>2$, then $f_{C}(\pi)_{[i, j]}$ is an interval only if it contains $[n-3]$. So the factor must have length at least $n-3$, meaning that $j \geqslant n-2$. Observe that $f_{C}(\pi)_{n-2}=\pi_{n-2}+1=n-1$ by Lemma 71 , so $f_{C}(\pi)_{[i, j]}$ must contain $[n-1]$. However, this is not the case since it omits $f_{C}(\pi)_{1}=\pi_{1}+1$ which is not $n$. So $j \leqslant 2$.

- Case 3: $i \geqslant 3$. Observe that $f_{C}(\pi)_{[i, j]}=\left(\pi_{i-1}+1\right)\left(\pi_{i}+1\right) \cdots\left(\pi_{j-1}+1\right)$. Since $\pi$ is simple, the factor $\pi_{[i-1, j-1]}$ is an interval only if $i \geqslant j$. So $i \geqslant j$.

Lemma 79. If $\sigma=(n-1) 1(n-2) \cdots n k \in A_{n}$ and $n \geqslant 6$, then $g_{A}(\sigma)$ is simple and avoids $P$. 
Proof. The mapping $g_{A}$ removes the first two entries and then reduces the result. Since $g_{A}(\sigma)$ is a reduced subsequence of $\sigma$, it avoids $P$.

Suppose that $g_{A}(\sigma)$ is not simple. Then there exists $[a, b] \subsetneq[3, n]$ and $[c, d] \subseteq[2, n]$ with $d \neq n-1$ and $b \geqslant a+2$ such that $\left\{\sigma_{i}: i \in[a, b]\right\}=[c, d]^{0}$ where $[c, d]^{0}:=$ $[c, d] \backslash\{n-1\}$.

If $d=n$ then $n-2 \in[c, d]^{0}$. Hence $a=3$ and $2 \in\left\{\sigma_{i}: i \in[a, b]\right\}=[c, d]^{0}$ which implies that $k=\pi_{n} \in[c, d]^{0}$ as well. But we have excluded the case $[a, b]=[3, n]$ since it is a trivial interval for $g_{A}(\sigma)$. This shows that $d \leqslant n-2$. But then $[c, d]^{0}=[c, d]$ and $\left\{\sigma_{i}: i \in[a, b]\right\}=[c, d]$ is an interval for $\sigma$.

Lemma 80. If $\sigma \in B_{n}$ and $n \geqslant 6$, then $g_{B}(\sigma)$ is simple and avoids $P$.

Proof. The mapping $g_{B}$ removes the first entry and the second last entry of $\sigma$. Since $g_{B}(\sigma)$ is a reduced subsequence of the permutation $\sigma$, it avoids $P$.

Suppose that $g_{B}(\pi)$ is not simple. Then there exists $[a, b] \subsetneq[2, n]$ and $[c, d] \subsetneq[1, n-2]$ with $b \neq n-1$ and $c<d$ such that $\left\{\pi_{i}: i \in[a, b]^{0}\right\}=[c, d]$ where $[a, b]^{0}:=[a, b] \backslash\{n-1\}$. If $b=n$ then $n-2 \in[a, b]^{0}$. Therefore $n-2=\pi(n-2) \in[c, d]$ and that implies that $(n-3) \in[c, d]$. Hence $a=2$ which means $[a, b]=[2, n]$. This contradiction implies that $b \leqslant n-2$. But then $[a, b]^{0}=[a, b]$ and $\pi_{[a, b]}=[c, d]$ is an interval for $\pi$.

Lemma 81. If $\sigma \in C_{n}$ and $n \geqslant 6$, then $g_{C}(\sigma)$ is simple and avoids $P$.

Proof. The mapping $g_{C}$ removes the second entry (which is 1 ) and then reduces the result. It is not hard to see that $g_{C}(\sigma)$ is a reduced subsequence of the permutation $\sigma$, so it must avoid $P$.

Suppose that $g_{C}(\pi)$ is not simple. Then there exists $[a, b] \subsetneq[1, n]$ and $[c, d] \subsetneq[2, n]$ with $a \neq 2$ and $d \geqslant c+2$ such that $\left\{\pi_{i}: i \in[a, b]^{0}\right\}=[c, d]$ where $[a, b]^{0}:=[a, b] \backslash\{2\}$.

If $a=1$ then $n-1 \in[c, d]$. Therefore $n-2$ or $n$ lies in $[c, d]$. Thus $b \geqslant n-3$ and this implies $2 \in \pi\left([a, b]^{0}\right)=[c, d]$. Therefore $k=\pi_{n} \in[c, d]$ and thus $b=n$, but we have excluded the case where $[a, b]=[1, n]$. This contradiction implies that $a \geqslant 3$. But then $[a, b]^{0}=[a, b]$ and $\pi_{[a, b]}=[c, d]$ is a nontrivial interval for $\pi$.

\subsection{Proof of Theorem 69}

It is well-known that there are no simple permutations of length 3 , so it is obvious that $A v_{3}^{S}(P)=0=F(0)$. For $n=4,5,6,7$, the values in Table 12 are easy to verify.

We proceed to prove the enumeration for $n \geqslant 6$ via induction. Recall that every simple $n$-permutation avoiding $P$ begins with $n-1$ by Lemma 70 . So for all $n \geqslant 6$, if $\pi \in A v_{n-2}^{S}(P)$ then we have

$$
\begin{aligned}
f_{A}\left(\pi_{1} \pi_{2} \cdots \pi_{n-2}\right)_{[1,3]} & =(n-1) 1\left(\pi_{1}+1\right) \\
& =(n-1) 1(n-2) \text { and } \\
f_{B}\left(\pi_{1} \pi_{2} \cdots \pi_{n-2}\right)_{[1,2]} & =(n-1) \pi_{1} \\
& =(n-1)(n-3),
\end{aligned}
$$




\begin{tabular}{|c|c|c|c|}
\hline & $\begin{array}{c}\boldsymbol{A}_{\boldsymbol{n}}:=\text { permutations } \\
\text { that start with } \\
(\boldsymbol{n}-\mathbf{1}) \mathbf{1}(\boldsymbol{n}-\mathbf{2})\end{array}$ & $\begin{array}{c}\boldsymbol{B}_{\boldsymbol{n}}:=\text { permutations } \\
\text { that start with } \\
(\boldsymbol{n}-\mathbf{1})(\boldsymbol{n}-\mathbf{3})\end{array}$ & $\begin{array}{c}\boldsymbol{C}_{\boldsymbol{n}}:=\text { permutations } \\
\text { that start with } \\
(\boldsymbol{n}-\mathbf{1}) \mathbf{1}(\boldsymbol{n}-\mathbf{3})\end{array}$ \\
\hline 4 & - & 3142 & - \\
5 & 41352 & - & - \\
6 & 514263 & 531462 & 6142573 \\
7 & 6152473 & 6413572 & 71524683 \\
\hline
\end{tabular}

Table 11: Simple $n$-permutations avoiding 2413, 3412, 3421 for $4 \leqslant n \leqslant 8$

\begin{tabular}{|c|c|c|c|c|c|}
\hline$n$ & $A v_{n}^{S}(P)$ & $A v_{n}^{S}(P)$ & $a_{n}$ & $b_{n}$ & $c_{n}$ \\
\hline 4 & 3142 & 1 & 0 & 1 & 0 \\
\hline 5 & 41352 & 1 & 1 & 0 & 0 \\
\hline 6 & $\begin{array}{l}514263 \\
531462\end{array}$ & 2 & 1 & 1 & 0 \\
\hline 7 & $\begin{array}{l}6152473, \\
6142573, \\
6413572\end{array}$ & 3 & 1 & 1 & 1 \\
\hline 8 & $\begin{array}{c}71625384, \\
71642583, \\
71524683, \\
75142683, \\
75314682\end{array}$ & 5 & 2 & 2 & 1 \\
\hline$k \geqslant 6$ & $A v_{k}^{S}(P)$ & $F(k-3)$ & $F(k-5)$ & $F(k-5)$ & $F(k-6)$ \\
\hline
\end{tabular}

Table 12: The number of simple permutations avoiding $2413,3412,3421$, where $4 \leqslant n \leqslant 8$ 
and for all $n \geqslant 6$ and $\pi \in B_{n-1}$ we have

$$
\begin{aligned}
f_{C}\left(\pi_{1} \pi_{2} \cdots \pi_{n-1}\right)_{[1,3]} & =(n-1) 1\left(\pi_{2}+1\right) \\
& =(n-1) 1(n-3) .
\end{aligned}
$$

Therefore, from Lemmas 76, 77 and 78,

$$
\begin{aligned}
\pi \in A v_{n-2}^{S}(P) & \Longrightarrow f_{A}(\pi) \in A_{n} \quad \text { and } \quad f_{B}(\pi) \in B_{n}, \\
\text { and } \pi \in B_{n-1} & \Longrightarrow f_{C}(\pi) \in C_{n},
\end{aligned}
$$

which means that $f_{A}\left(A v_{n-2}^{S}(P)\right) \subseteq A_{n}, f_{B}\left(A v_{n-2}^{S}(P)\right) \subseteq B_{n}$ and $f_{C}\left(B_{n-1}^{S}(P)\right) \subseteq C_{n}$.

On the other hand, Lemmas 79,80 and 81 clearly show that

$$
\begin{aligned}
& \sigma \in A_{n} \Longrightarrow g_{A}(\sigma) \in A v_{n-2}^{S}(P) \text { and } \\
& \sigma \in B_{n} \Longrightarrow g_{B}(\sigma) \in A v_{n-2}^{S}(P) \text { and } \\
& \sigma \in C_{n} \Longrightarrow g_{C}(\sigma) \in B_{n-1} \text { and }
\end{aligned}
$$

which means that $g_{A}\left(A_{n}\right) \subseteq A v_{n}^{S}(P), g_{B}\left(B_{n}\right) \subseteq A v_{n}^{S}(P)$ and $g_{C}\left(C_{n}\right) \subseteq B_{n-1}$. Therefore, $g_{A}, g_{B}$ and $g_{C}$ is in fact the inverse of $f_{A}, f_{B}$ and $f_{C}$, respectively. Moreover, we claim that for all $n \geqslant 6$,

$$
a_{n}=\left|A v_{n-2}^{S}(P)\right|, \quad b_{n}=\left|A v_{n-2}^{S}(P)\right|, \quad \text { and } \quad c_{n}=b_{n-1} .
$$

Suppose that for some $k \geqslant 6$,

$$
a_{\ell}=F(\ell-5), \quad b_{\ell}=F(\ell-5), \quad \text { and } \quad c_{\ell}=F(\ell-6)
$$

for all $\ell \in\{4,5, \ldots, k-1\}$. Then from the statements above,

$$
\begin{aligned}
a_{k} & =\left|A v_{k-2}^{S}(P)\right| \\
& =a_{k-2}+b_{k-2}+c_{k-2} \\
& =F(k-7)+F(k-7)+F(k-8) \\
& =F(k-7)+F(k-6) \\
& =F(k-5), \\
\text { so } \quad b_{k}=\left|A v_{k-2}^{S}(P)\right| & =F(k-5) \quad \text { and } \quad c_{k}=b_{k-1}=F(k-6) .
\end{aligned}
$$

So the claim is true by induction. Therefore, for all $n \geqslant 6$,

$$
\begin{aligned}
\left|A v_{n}^{S}(P)\right| & =a_{n}+b_{n}+c_{n} \\
& =F(n-5)+F(n-5)+F(n-6) \\
& =F(n-5)+F(n-4) \\
& =F(n-3) .
\end{aligned}
$$

This concludes the proof of Theorem 69 . 


\section{Summary}

In this paper, we elucidated connections between the avoidance sets of some POPs and other combinatorial objects by constructing explicit bijections between the relevant sets, as a direct response to five of the 15 open questions posed by Gao and Kitaev [5]. These bijections were derived primarily by analysing the simple permutations of the avoidance sets and how the rest of the set could be obtained from their inflations. This was made possible by illustrating the permutation matrices as lattice matrices, which is a novel concept introduced in this paper. The bijections constructed in this paper are a testament to the fundamental role that simple permutations play in the study of pattern-avoiding permutations. It also demonstrates the intricate connections that avoidance sets of POPs have with many other combinatorial objects, and provides a way to relate seemingly disparate combinatorial objects through their connections to the family of POPs. We also enumerated the number of simple $n$-permutations avoiding the patterns 2413, 3412 and 3421 for all $n$, giving a concrete example of an avoidance set with a finite basis and infinitely many simple permutations.

\section{Further Work}

The remaining ten questions posed by Gao and Kitaev [5] remain open. Given the bijections we have constructed, it would be interesting to know whether they can be generalized further, by studying generalizations of the POPs or of the combinatorial objects. The following questions are natural extensions of the problem that was discussed in Section 4:

1. Are there any combinatorial objects that have a natural bijective relationship with the avoidance set of $P_{k}$ for any $k \geqslant 5$ ?

2. Is there a POP whose avoidance set is in bijection with the levels in compositions of ones, twos and threes?

3. Are there any combinatorial objects that have a natural bijective relationship with the avoidance set of the POP with $k$ elements labelled $1,2, \ldots, k$ where $1>3>5$, or, more generally, with $1>3>\cdots>2 i+1$ for some $i \geqslant 2$ ?

The enumeration of $A v_{n}\left(R_{k}\right)$ for $k \geqslant 6, n \geqslant 1$, where $R_{k}$ is defined in Section 5, is an open question. It could also be interesting to enumerate $\mathfrak{S}_{k, n}$, which we define as the set of permutations whose partial sums of signed displacements do not exceed $k$, for all $k \geqslant 3$, and check if there exist any $k$ and $\ell$ such that $\left|\mathfrak{S}_{k, n}\right|=\left|A v_{n}\left(R_{\ell}\right)\right|$ for all $n \geqslant 1$. Finally, Gao and Kitaev [5] observed that sequence $\left|A v_{n-1}\left(R_{4}\right)\right|_{n \geqslant 2}$ corresponds to sequence A232164 as well. The latter sequence counts the number of Weyl group elements, not containing an $s_{r}$ factor, which contribute nonzero terms to Kostant's weight multiplicity formula when computing the multiplicity of the zero-weight in the adjoint representation for the Lie algebra of type $C$ and rank $n$. Using our analysis on the set $A v\left(R_{4}\right)$, one may be able to construct a natural bijection between these two sets more easily. 
During our study of the simple permutations that avoid the patterns 2413, 3412 and 3421, we discovered using the PermLab software that the addition of the pattern 2431 to the basis does not change the set of simple permutations for small $n$. It can be proved that the simple permutations constructed by the recursive functions to build the set $A v_{n}^{S}(2413,3412,3421)$ indeed avoid 2431. This observation leads us to an interesting question: Which avoidance sets have the same set of simple permutations?

\section{References}

[1] M. Albert. PermLab. 2012. http://www.cs.otago.ac.nz/PermLab/

[2] M.H. Albert and M.D. Atkinson. Simple Permutations and Pattern Restricted Permutations. Discrete Mathematics 300. 1-3 (2005), pp. 1-15.

[3] J. Buhler, D. Eisenbud, R. Graham and C. Wright. Juggling Drops and Descents. The American Mathematical Monthly 101.6 (1994), pp. 507-519. ISSN : 00029890, 19300972. http://www. jstor.org/stable/2975316.

[4] F. Chung and R. Graham. Primitive Juggling Sequences. The American Mathematical Monthly 115.3 (2008), pp. 185-194. http://www.jstor.com/stable/27642443.

[5] A. Gao and S. Kitaev. On Partially Ordered Patterns of Length 4 and 5 in Permutations. The Electronic Journal Of Combinatorics 26(3) (2019) \#P3.26 https://doi .org/10.37236/8605.

[6] S. Kitaev. Patterns in Permutations and Words. Springer Berlin Heidelberg, 2011. https://doi .org/10.1007/978-3-642-17333-2.

[7] J.K. Percus. Combinatorial Methods, Applied Mathematical Sciences \#4. New York: Springer-Verlag, 1971. ISBN : 978-0-387-90027-8.

[8] G. Pogosyan, I. G. Rosenberg, A. Nozaki and M. Miyakawa. The number of orthogonal permutations. European Journal of Combinatorics 16.1 (1995), pp. 71-85. https : //doi .org/10.1016/0195-6698(95)90091-8

[9] N. J. A. Sloane. The Online Encyclopedia of Integer Sequences. https://oeis.org 